\title{
A Blended Soundproof-to-Compressible Numerical Model for Small- to Mesoscale Atmospheric Dynamics
}

\author{
Tommaso Benacchio, WARren P. O’Neill, AND Rupert Klein \\ FB Mathematik \& Informatik, Freie Universität Berlin, Berlin, Germany
}

(Manuscript received 26 November 2013, in final form 20 June 2014)

\begin{abstract}
A blended model for atmospheric flow simulations is introduced that enables seamless transition from fully compressible to pseudo-incompressible dynamics. The model equations are written in nonperturbation form and integrated using a well-balanced second-order finite-volume discretization. The semi-implicit scheme combines an explicit predictor for advection with elliptic corrections for the pressure field. Compressibility is implemented in the elliptic equations through a diagonal term. The compressible/pseudo-incompressible transition is realized by suitably weighting the term and provides a mechanism for removing unwanted acoustic imbalances in compressible runs.

As the gradient of the pressure is used instead of the Exner pressure in the momentum equation, the influence of perturbation pressure on buoyancy must be included to ensure thermodynamic consistency. With this effect included, the thermodynamically consistent model is equivalent to Durran's original pseudoincompressible model, which uses the Exner pressure.

Numerical experiments demonstrate quadratic convergence and competitive solution quality for several benchmarks. With the inclusion of an additional buoyancy term required for thermodynamic consistency, the " $p-\rho$ formulation" of the pseudo-incompressible model closely reproduces the compressible results.

The proposed unified approach offers a framework for models that are largely free of the biases that can arise when different discretizations are used. With data assimilation applications in mind, the seamless compressible/pseudo-incompressible transition mechanism is also shown to enable the flattening of acoustic imbalances in initial data for which balanced pressure distributions are unknown.
\end{abstract}

\section{Introduction}

Physical processes in the atmosphere feature a wide range of spatiotemporal scales described by the fully compressible nonhydrostatic flow equations. Accordingly, nonhydrostatic fully compressible modeling approaches hold sway in atmospheric research codes and in operational dynamical cores; for example, Icosahedral Nonhydrostatic model (ICON; Zängl et al. 2014), Nonhydrostatic Unified Model of the Atmosphere (NUMA; Kelly and Giraldo 2012), (DUNE; Brdar et al. 2013), the models in use at the National Center for Atmospheric Research (NCAR; Wong et al. 2014), the European Centre for Medium-Range Weather Forecasts (ECMWF; Hortal 2002; Smolarkiewicz et al. 2014), the Met Office (Davies et al. 2005; Wood et al. 2014), and others.

Corresponding author address: Tommaso Benacchio, Met Office, FitzRoy Road, Exeter EX1 3PB, United Kingdom.

E-mail: tommaso.benacchio@metoffice.gov.uk
Despite very successful ongoing developments, the proper treatment of multiple characteristic time scales in atmospheric simulations remains a matter of scientific research. Two of the biggest obstacles of multiplescale simulations are (i) the discretization of fast processes in the governing equations and (ii) balanced data assimilation.

Numerical stiffness is the source of the first remaining obstacle. Except for inertia-gravity waves of long wavelength, which are not considered here, quantities of meteorological interest propagate at low speed compared with sound waves. Sound modes are said to be nearly balanced and their effects are considered negligible for atmospheric dynamics. The difference between the sound and flow speeds stiffens the numerics of fully compressible solvers rendering straightforward explicit schemes impractical because of severe stability-related time step constraints.

Filtering the data with respect to fast modes while minimally distorting the ensuing dynamics is the second remaining obstacle. Computational simulations never 
exactly track the evolution of the considered system. Hence, data assimilation is needed for exploiting observational data at regular time intervals to set up initial data for the next simulation period. However, importing observed field data from local weather stations directly to adjacent grid points would disregard the aforementioned balances of the fast modes. For example, in the presence of a low pressure system in the summer with high levels of convection, the local vertical velocities would project onto nonhydrostatic and compressible modes yielding strongly unbalanced data on the numerical grid. Efficiently controlling such modes remains a challenge in data assimilation.

Numerical approaches aimed at overcoming the stiffness are split-explicit, semi-implicit, and fully implicit numerical time integrators for the fully compressible flow equations. The first class of schemes subcycles a simplified discretization of the fast wave processes at short time steps and employs suitable synchronization procedures for coupling the results to large time steps of the slower modes (Skamarock and Klemp 1994, 2008; Jebens et al. 2009). Another option would be to adopt a fully implicit approach that even overcomes the time step restrictions associated with explicit discretizations of advection. Because of their computational expense these schemes have, to our knowledge, thus far not found widespread application in meteorology. A notable exception is the work by Reisner et al. (2005).

The focus of the present work lies instead on semiimplicit discretizations that invoke implicit integrators for the terms in the equations representing the fast wave modes while treating the slow modes explicitly. Many approaches to semi-implicit discretization for atmospheric flows have been reported (e.g., Bonaventura 2000; Gatti-Bono and Colella 2006; Restelli and Giraldo 2009; Jebens et al. 2011; Durran and Blossey 2012; Giraldo et al. 2013; Wood et al. 2014; Smolarkiewicz et al. 2014; Weller and Shahrokhi 2014). For all-speed flow discretizations in computational fluid dynamics the reader is referred to Casulli and Greenspan (1984), Bijl and Wesseling (1998), Munz et al. (2003), and Kwatra et al. (2009).

An alternative to these numerical approaches to overcoming the stiffness is to adopt a "soundproof" model. These reduced dynamical models include a diagnostic constraint on the velocity divergence and therefore do not support sound waves. The divergence constraint needs to be maintained numerically, which entails the solution of an elliptic pressure equation. Soundproof models suitable for atmospheric motions covering vertical distances comparable to the pressure scale height are the anelastic (Lipps and Hemler 1982; Bannon 1996) and pseudo-incompressible models (Durran 1989; Klein and Pauluis 2012).
Soundproof models have successfully been used to simulate small to mesoscale flows, and their validity as slow-flow limit models has recently been established on theoretical grounds (Klein et al. 2010; Achatz et al. 2010). However, their applicability to large-scale motions is still under debate (Davies et al. 2003; Dukowicz 2013) despite recent successful large-scale simulations for atmospheric (Smolarkiewicz and Dörnbrack 2008; Smolarkiewicz et al. 2014) and astrophysical (Nonaka et al. 2010; Smolarkiewicz and Charbonneau 2013) applications.

In line with these observations, one of our goals is to develop a numerical scheme for the fully compressible equations that defaults to the pseudo-incompressible limit for slow flows on small- to mesoscales. Such asymptotically adaptive schemes have a substantial history of studies (Klein 2000; Klein et al. 2001; Gatti-Bono and Colella 2006; Cullen 2007; Haack et al. 2012) in which the low Mach or low Froude number limits are discretely recovered through careful identification and separate discretization of the advection, acoustic, and/or buoyancy terms in the fully compressible equations. In the present work we suggest a particularly straightforward approach of this type that is directly motivated by the theoretical framework set out in Klein $(2009,2010)$.

More specifically, this paper documents the construction of a semi-implicit second-order accurate numerical method for the simulation of weakly compressible atmospheric flows that shares the principal components of the discretization with the soundproof solver by Klein (2009). The time integration for the fully compressible equations derives from that of the pseudo-incompressible model and the required adjustments amount to no more than adding a diagonal term to the matrix of the elliptic pressure problem and synchronizing the cellcentered and node-based pressures. This is similar in spirit to parallel developments by Smolarkiewicz et al. (2014) but technically different. In particular, these authors do not address the possibility of a seamless blending of models and they work with perturbation variables and with the Exner pressure in the momentum equation.

Besides constructing the compressible flow solver, we design the discretization such that it can be used directly to solve a continuous family of weakly compressible models that interpolate seamlessly between the fully compressible and pseudo-incompressible ones. This is realized by exploiting the close structural similarity of these two limiting models when written in conservative, nonperturbation form for the densities of mass, momentum, and potential temperature.

In the context of increasing computing resources and ever smaller scales accessible in high-resolution weather and climate simulations, it is of arguable interest to 
operate different analytical formulations within a single numerical framework. Such a unified numerical scheme becomes all the more desirable in the light of a recent study (Smolarkiewicz and Dörnbrack 2008) that compared the errors made by using different numerical methods for the same model equations with those made by considering different equation systems discretized with nearly identical numerics. These authors found, somewhat surprisingly, that the former errors exceeded the latter, and this underlines the importance of comparing flow models within one and the same numerical framework. In an interesting investigation of this type, Smith and Bannon (2008) compared anelastic and compressible models in a case of localized instantaneous diabatic warming.

A second motivation for implementing the seamless model family lies in its potential use for balanced data assimilation. By adjusting the model interpolation parameter accordingly from zero to unity, such a "blended" scheme can be tuned to perform a few time steps in pseudo-incompressible mode and to then transition to its fully compressible mode after a few further steps. As we will show, this effectively reduces initial acoustic imbalances. Considering the factors affecting predictability of the simulated precipitation field in cloud-resolving models, Hohenegger and Schär (2007) showed that uncontrolled small-scale acoustic perturbations may contribute to rapid error growth at the mesoscale.

The scheme we propose has more potentially attractive features. One of these features is the formulation in a nonperturbation form that does not rely on subtraction of a background state for accuracy. This is achieved for the present collocated finite-volume method by a well-balanced discretization of the pressure gradient and gravity terms following Botta et al. (2004) and Klein (2009). Moreover, the scheme uses the gradient of the thermodynamic instead of the Exner pressure, thereby allowing for a conservative discretization of the momentum flux induced by the pressure force. In addition, as pointed out by Klein and Pauluis (2012), Durran's original formulation of the pseudo-incompressible model using Exner pressure cannot be easily extended to general equations of state. One step toward overcoming this obstacle is to adopt a formulation with pressure instead of Exner pressure in the momentum equation as done in this paper. Yet, this formulation is thermodynamically consistent only if first-order density perturbations are included in the gravity term in addition to Durran's "pseudodensity." For an ideal gas with constant specific heat capacities, Durran's model and the present thermodynamically consistent formulation are equivalent as a short calculation using the transformations $\pi_{0}=\left(p_{0} / p_{\text {ref }}\right)^{R / c_{p}}$ and $\pi^{\prime}=p^{\prime} /\left(c_{p} P_{0}\right)$ shows. A second step that is also necessary in extending to general equations of state, but which is not pursued here, is a reformulation of the velocity divergence constraint. This step is needed because in this case the pressure equation can no longer be easily cast into a simple conservation law (Almgren et al. 2006a,b; Klein and Pauluis 2012).

Furthermore, the transition from the pseudoincompressible via the blending to the compressible model is achieved by minimal code adjustments. These involve reassigning certain weights in the grid stencil of the elliptic correction equations and applying a weighted superposition of pressure updates. These updates are calculated from the elliptic equations and from the conservative balance of potential temperature.

The paper is structured as follows. Compressible, pseudo-incompressible, and blended models are presented in section 2. Section 3 summarizes the numerics. The results of numerical simulations in a number of twodimensional test cases are documented in section 4. Grid convergence with the expected second-order rate is verified in a benchmark involving advection of a smooth axisymmetric vortex. For the standard test cases of a rising hot air thermal, density current, and inertia-gravity waves, we compare the predictions obtained with the compressible and pseudo-incompressible models and demonstrate the importance of the thermodynamic consistency correction within the pseudo-incompressible framework. Usage of the blended model for filtering acoustic imbalances is demonstrated for both short sound-resolving time steps and for time steps corresponding to an advective Courant-Friedrichs-Lewy (CFL) number of order unity. Section 5 provides the conclusions and an outline of open issues and future work.

\section{Theoretical framework}

\section{a. Fully compressible equations}

The dry, inviscid fully compressible equations (hereafter referred to as FC), describe conservation of mass, momentum, and energy under the influence of gravity. If we neglect rotational effects and use the transport equation for potential temperature to describe the energy balance, they read in conservative form and in the dry adiabatic case:

$$
\begin{gathered}
\frac{\partial \rho}{\partial t}+\nabla \cdot(\rho \mathbf{v})=0 \\
\frac{\partial \rho \mathbf{v}}{\partial t}+\nabla \cdot(\rho \mathbf{v} \circ \mathbf{v}+p \mathbf{l})=-\rho g \mathbf{k}, \\
\frac{\partial P}{\partial t}+\nabla \cdot(P \mathbf{v})=0 .
\end{gathered}
$$


Here, $\rho$ denotes the fluid density, $\mathbf{v}$ is the velocity vector, $\circ$ is the tensor product, $g$ is the acceleration of gravity, $\mathbf{k}$ is the vertical unit vector, and $\mathbf{I}$ is the identity tensor. As in Klein (2009), we have introduced the equation of state:

$$
P=\rho \theta=\frac{p_{\text {ref }}}{R}\left(\frac{p}{p_{\text {ref }}}\right)^{1 / \gamma}
$$

where potential temperature is defined as

$$
\theta=T\left(\frac{p}{p_{\text {ref }}}\right)^{(1-\gamma) / \gamma} \text { and } T=\frac{p}{\rho R}
$$

is the temperature. The $R$ is the gas constant for dry air and $\gamma$ is the isentropic exponent. Hereafter, we take $\gamma=1.4$ and $R=287 \mathrm{~N} \mathrm{~m} \mathrm{~kg}^{-1} \mathrm{~K}^{-1}$ throughout. For smooth flows, (1c) can equivalently replace total energy conservation in a finite-volume discretization, which is common in numerical meteorology, but which would not be adequate for flows with shocks (LeVeque 2002). Together, (1a) and (1c) describe mass conservation and the advection of potential temperature, while (1c) is equivalent to the pressure evolution equation $p_{t}+\mathbf{v} \cdot \nabla p+$ $\gamma p \nabla \cdot \mathbf{v}=0$. Thus, a discretization of (1c) directly controls the pressure evolution, and this is central to the blended compressible-soundproof formulation to be presented below.

The system is closed by appropriate initial and boundary conditions which we will specify in conjunction with specific test cases below.

For later reference, using (2), we compute

$$
\frac{\partial P}{\partial p}=\frac{1}{R \gamma}\left(\frac{p}{p_{\text {ref }}}\right)^{(1 / \gamma)-1}=\frac{1}{R \gamma}\left(\frac{P R}{p_{\text {ref }}}\right)^{1-\gamma} .
$$

\section{b. The pseudo-incompressible approximation}

The pseudo-incompressible model (Durran 1989) is commonly derived from a compressible model that formulates the pressure gradient term in the momentum equation using the Exner pressure:

$$
\pi=\left(\frac{p}{p_{\text {ref }}}\right)^{(\gamma-1) / \gamma}
$$

so that, in view of (3), one finds

$$
\frac{1}{\rho} \nabla p \equiv c_{p} \theta \nabla \pi
$$

To retain flexibility of the developed code, in particular with respect to generalizations of the equation of state, we adopt the $p-\rho$ formulation here (Klein and Pauluis 2012). When written in the latter form, extra care must be taken in formulating the momentum equation to ensure that it retains the influences of the pressure perturbation up to first order.

As in Durran (1989) we start our derivations by assuming that the pressure does not vary much from its hydrostatic background value and can be written as

$$
p=p_{0}(z)+p^{\prime}(\mathbf{x}, t),
$$

where $p^{\prime} / p_{0} \ll 1$ and

$$
\frac{\partial p_{0}}{\partial z}=-\rho_{0} g
$$

Using (7) in the equation of state in (2) gives, with a Taylor expansion:

$$
\begin{aligned}
\rho & =\frac{1}{\theta} \frac{p_{\text {ref }}}{R}\left(\frac{p_{0}+p^{\prime}}{p_{\text {ref }}}\right)^{1 / \gamma} \approx \frac{1}{\theta} \frac{p_{\text {ref }}}{R}\left(\frac{p_{0}}{p_{\text {ref }}}\right)^{1 / \gamma}\left(1+\frac{p^{\prime}}{\gamma p_{0}}\right) \\
& =\rho^{*}\left(1+\frac{p^{\prime}}{\gamma p_{0}}\right),
\end{aligned}
$$

where $\rho^{*}$ is called the pseudodensity and is defined as the density calculated at the background pressure but using the full potential temperature:

$$
\rho^{*}=\frac{1}{\theta} \frac{p_{\text {ref }}}{R}\left(\frac{p_{0}}{p_{\text {ref }}}\right)^{1 / \gamma}=\rho\left(p_{0}, \theta\right) .
$$

To filter sound waves we suppress the effect of pressure perturbations on density to obtain

$$
\left(\rho^{*}\right)_{t}+\nabla \cdot\left(\rho^{*} \mathbf{v}\right)=0 .
$$

However, in the momentum equation we want to keep the effect of the pressure perturbations up to first order. Using an expansion as in (10) we rewrite (1b) in nonconservative form

$\mathbf{v}_{t}+(\mathbf{v} \cdot \nabla) \mathbf{v}+\frac{1}{\rho^{*}}\left(1-\frac{p^{\prime}}{\gamma p_{0}}\right) \nabla\left(p_{0}+p^{\prime}\right)=-g \mathbf{k}$.

Keeping terms in (12) up to first order in the pressure perturbation and rearranging we get

$\mathbf{v}_{t}+(\mathbf{v} \cdot \nabla) \mathbf{v}+\frac{1}{\rho^{*}} \nabla\left(p_{0}+p^{\prime}\right)=-\left(1+\frac{1}{\rho^{*}} \frac{\rho_{0}}{\gamma p_{0}} p^{\prime}\right) g \mathbf{k}$.

We rewrite (13) in conservative form by multiplying by $\rho^{*}$ and using (11): 
$\left(\rho^{*} \mathbf{v}\right)_{t}+\nabla \cdot\left(\rho^{*} \mathbf{v} \circ \mathbf{v}\right)+\nabla p=-\left(\rho^{*}+\frac{\rho_{0}}{\gamma p_{0}} p^{\prime}\right) g \mathbf{k}$.

Last, we redefine $P$ as

$$
P \approx \rho^{*} \theta=\frac{p_{\text {ref }}}{R}\left(\frac{p_{0}}{p_{\text {ref }}}\right)^{1 / \gamma}=P_{0}
$$

and (1c) becomes

$$
\left(P_{0}\right)_{t}+\nabla \cdot\left(P_{0} \mathbf{v}\right)=\nabla \cdot\left(P_{0} \mathbf{v}\right)=0 .
$$

In (16) we have used that $P$ is now a function of $p_{0}$ only, which allows us to drop the time derivative term and the evolution equation becomes a divergence constraint. This constraint enforces the pseudo-incompressible form of the density equation in (11) thereby filtering the effect of pressure perturbations on the density and thus filtering sound waves.

The complete pseudo-incompressible governing equations are given by

$$
\begin{aligned}
\left(\rho^{*}\right)_{t}+\nabla \cdot\left(\rho^{*} \mathbf{v}\right) & =0 \\
\left(\rho^{*} \mathbf{v}\right)_{t}+\nabla \cdot\left(\rho^{*} \mathbf{v} \circ \mathbf{v}\right)+\nabla p & =-\left(\rho^{*}+\frac{\rho_{0}}{\gamma p_{0}} p^{\prime}\right) g \mathbf{k} \\
\nabla \cdot\left(P_{0} \mathbf{v}\right) & =0 .
\end{aligned}
$$

Klein (2009) showed agreement between (17a)-(17c) and the original formulation of Durran (1989) to leading and first order in a perturbation expansion for small pressure variations. Moreover, if Exner pressure variables are introduced so that $\pi_{0}=\left(p_{0} / p_{\text {ref }}\right)^{R / c_{p}}$ and $\pi^{\prime}=$ $p^{\prime} /\left(c_{p} P_{0}\right)$, a straightforward calculation shows that the original formulation of Durran (1989) and the present $\mathrm{PI}_{\rho, p}^{\mathrm{tc}}$ formulation are actually equivalent at the level of the partial differential equations. An advantage of our formulation is that it is more easily extended to incorporate more complex equations of state and that it is "thermodynamically consistent." This notion refers to the existence of well-defined thermodynamic potentials describing the proper increase-decrease of an entropy variable in the diabatic case (Klein and Pauluis 2012). Note, however, that completing the extension to general equations of state also requires a reformulation of the divergence constraint (Almgren et al. 2006a,b; Klein and Pauluis 2012).

\section{c. A blended compressible/pseudo-incompressible model}

In Klein (2009) the task of incorporating the time derivative term in (1c) and modeling the fully compressible
TABLE 1. Model configurations used in the numerical scheme.

\begin{tabular}{lcc}
\hline \multicolumn{1}{c}{ Model name } & Abbreviation & $(\alpha, \beta)$ \\
\hline Fully compressible & $\mathrm{FC}$ & $(1,0)$ \\
$\begin{array}{c}\text { Thermodynamic consistent } \\
\text { pseudo-incompressible }\end{array}$ & $\mathrm{PI}_{\rho, p}^{\mathrm{tc}}$ & $(0,1)$ \\
$\begin{array}{c}\text { Nonthermodynamic consistent } \\
\text { pseudo-incompressible }\end{array}$ & $\mathrm{PI}_{\rho, p}$ & $(0,0)$ \\
\hline
\end{tabular}

dynamics was left for future work. Here we aim to merge the compressible, pseudo-incompressible, and thermodynamically consistent discretizations in the " $p-\rho$ formulation" for the momentum equation in a single numerical model featuring

- a conservative discretization with respect to $\rho, \rho \mathbf{v}$, $\rho \theta \equiv P$,

- second-order accuracy,

- time steps independent of the sound speed,

- a continuous transition between pseudo-incompressible and compressible forms, and

- a well-balanced discretization that does not rely on subtraction of a background state.

The blended equations are given as follows, for $\alpha \in$ $\{0,1\}$ :

$$
\rho_{t}+\nabla \cdot(\rho \mathbf{v})=0
$$

$$
\begin{gathered}
(\rho \mathbf{v})_{t}+\nabla \cdot(\rho \mathbf{v} \circ \mathbf{v})+\nabla p=-g \mathbf{k}\left[\rho+(1-\alpha) \beta \frac{\rho_{0}}{\gamma p_{0}} p^{\prime}\right] \\
\alpha P_{t}+\nabla \cdot(P \mathbf{v})=0
\end{gathered}
$$

For $\alpha=0$, the two pseudo-incompressible models with the $p-\rho$ formulation of the pressure gradient term are retrieved. Then, setting $\beta=1$ selects the thermodynamically consistent $\left(\mathrm{PI}_{\rho, p}^{\mathrm{tc}}\right)$ model whereas setting $\beta=0$ retrieves the "naive" pseudo-incompressible $\left(\mathrm{PI}_{\rho, p}\right)$ model. We note that in $\mathrm{PI}_{\rho, p}$ and $\mathrm{PI}_{\rho, p}^{\mathrm{tc}}$ the density $\rho$ takes the role of the pseudodensity, which was denoted by $\rho^{*}$ in (17b), and necessitates the additional term for thermodynamic consistency in the momentum equation in (18b) for $(\alpha, \beta)=(0,1)$. As the model parameter $\alpha$ is adjusted from 0 to 1 , the effect of pressure perturbations on density is retrieved in a continuous fashion. This formulation recovers the fully compressible (FC) dynamics for $\alpha=1$. A summary of the model configurations is given in Table 1 .

The system in (18) features nonapproximate mass and momentum equations for $\alpha \in\{0,1\}$ when $\beta=1$. The reason is that the $\mathrm{PI}_{\rho, p}^{\mathrm{tc}}$ model is equivalent to Durran's original pseudo-incompressible model with the 
" $\pi-\theta$ formulation" of the pressure gradient term. Klein et al. (2014) observe that the model satisfies an energy conservation law with a definition of the total energy that is an interpolation between those of the fully compressible and the pseudo-incompressible models. The model's internal wave dispersion properties for realistic stratifications are close to those of the limiting models. This follows from related analyses for the limiting models by Klein (2010) and the fact that the underlying SturmLiouville problems depend smoothly on the defining data. We also refer to Vasil et al. (2013) for related analysis and relegate further discussion to a future publication.

In (18) the $\alpha$ and $\beta$ parameters are introduced to formulate the $\mathrm{FC}, \mathrm{PI}_{\rho, p}^{\text {tc }}$, and $\mathrm{PI}_{\rho, p}$ models conveniently in one and the same set of equations. Only discrete values $\alpha, \beta \in\{0,1\}$ make sense to begin with. Yet, let us consider the resulting model equations for any $\alpha \in[0,1]$. A seamless discretization that allows integration of (18) for any of these values can be used to our advantage in some meteorologically interesting situation.

Suppose we are to initialize one of the well-known test cases of a rising warm-air bubble or flow over a mountain. As in "real meteorology," we are not interested in acoustic perturbations and would like to simulate acoustically balanced flows. Yet, we have no analytical way to determine the balanced pressure distributions that would be associated with given initial data for potential temperature and velocity.

However, knowing that the pseudo-incompressible models provide good approximations to compressible flows free of sound waves, we can attempt to generate reasonable approximations to the missing pressure fields by starting a simulation pseudo-incompressibly with $\alpha=0$ for, say, $S_{1}$ time steps. Within the next $S_{2}$ time steps we increase $\alpha$ continuously from 0 to 1 , and after time step $S_{1}+S_{2}$ we maintain $\alpha=1$ to operate the model in fully compressible mode. This procedure should generate a compressible flow simulation that is balanced with respect to acoustic modes essentially from the start. Promising related results for the rising bubble test are discussed in section 4 below.

We conjecture that such a smooth blending of balanced and unbalanced model equations within a common discretization framework could substantially contribute to resolving similar balancing issues in the context of data assimilation.

\section{Numerical framework}

A semi-implicit finite-volume method is used to approximate the dynamics of the blended model. The scheme is a variant and extension of the soundproof solver described in Klein (2009). An outline is presented

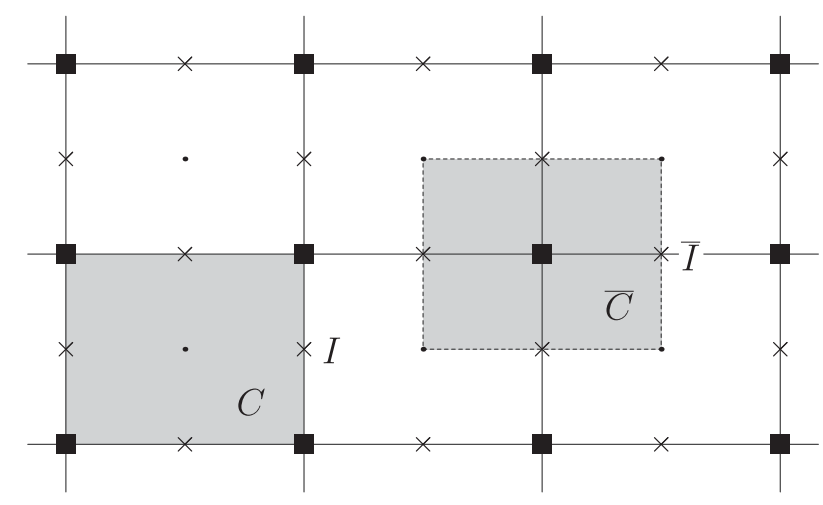

FIG. 1. Computational grid for the numerical scheme. Solid lines define cells and dashed lines define dual cells, used for the second correction. Dots, squares, and crosses denote cell centers, nodes, and interface centers, respectively.

here, for more details see the appendix. The discrete solution of (18) is obtained by the following time stepping procedure, say from $t^{n}$ to $t^{n+1}$ :

- An explicit predictor solves an auxiliary hyperbolic system obtained by replacing the pressure gradient in the momentum equation in (18b) with its value at time level $t^{n}$. This step yields second-order accurate $\rho, \theta$, and $P$.

- A first elliptic corrector solves for the cell-centered pressure time increment $\delta p=p^{n+1}-p^{n}$ by enforcing consistency with the pressure equation in (18c). This step also corrects the advecting fluxes in (18a) and (18b).

- The solution of a second elliptic problem is used to correct the pressure-related momentum flux for fully second-order accurate updates of the cell-centered momenta.

For the time discretization we divide the simulation time interval $[0, T]$ into $N$ subintervals, with $t_{0}=0, t^{n+1}=$ $t^{n}+(\Delta t)^{n}$ for $n=0,1, \ldots, N-1$. For any variable $X$, we denote $X^{n}=X\left(t^{n}\right)$ and $(\Delta t)^{n}=O(T / N)$ denotes the time steps. In the implementation, a dynamically adaptive choice of the time step based on fixing the Courant number is implemented, see the appendix for details. The spatial domain is divided into primary computational cells $C_{i, j}$ (finite volumes) with $i=1, \ldots, \mathcal{N}_{x}, j=1, \ldots, \mathcal{N}_{z}$, in two dimensions according to a Cartesian grid arrangement. The cells $C_{i, j}$ are separated by interfaces $I_{i+1 / 2, j}, I_{i, j+1 / 2}$ as shown in Fig. 1 . The extension to three dimensions is straightforward. The primary variables $\rho, \rho \mathbf{v}, P$ are stored at the centers of the primary cells $C_{i, j}$. Pressures are computed at centers of the primary cells $C_{i, j}$ in the first correction step and at the centers of the dual cells $\bar{C}_{i+1 / 2, j+1 / 2}$ shown in Fig. 1 in the second correction step. 


\section{a. Step 1: Predictor}

In the first substep for a full time step $t^{n} \rightarrow t^{n+1}$, the following auxiliary hyperbolic system, obtained from (18) by freezing $p$ and $p^{\prime}$ at time level $t^{n}$, is solved (Klein 2009):

$$
\begin{gathered}
\frac{\partial \rho}{\partial t}+\nabla \cdot(\rho \mathbf{v})=0 \\
\frac{\partial \rho \mathbf{v}}{\partial t}+\nabla \cdot\left(\rho \mathbf{v} \circ \mathbf{v}+p^{n} \mathbf{I}\right)=-g \mathbf{k}\left[\rho+(1-\alpha) \beta \frac{\rho_{0}}{\gamma p_{0}}\left(p^{\prime}\right)^{n}\right]
\end{gathered}
$$

$$
\frac{\partial P}{\partial t}+\nabla \cdot(P \mathbf{v})=0
$$

A two-stage strong stability-preserving Runge-Kutta method (Gottlieb et al. 2001) is used for time integration here [Klein (2009) instead used a Monotonic Upstreamcentered Scheme for Conservation Laws (MUSCL) technique and directional operator splitting]. The spatial discretization at any stage of the Runge-Kutta time integrator is performed with a finite-volume approach. That is, discrete variables $X_{C}, X=\rho, \rho \mathbf{v}$, and $P$, are defined as approximations of the cell averages set at the cell centers:

$$
X_{C}=\frac{1}{|C|} \int_{C} X d x+O\left(\Delta x^{2}\right)
$$

where $|C|$ is the cell volume. To achieve second-order accuracy in space, piecewise linear reconstruction of $P, \mathbf{v}$, and the advected quantities $(1 / \theta, \mathbf{v} / \theta)$ is applied within the grid cells. The reconstructed values are used to determine any data required at the cell interfaces and to evaluate the numerical flux functions. The pressure variables $p^{n}$ and $\left(p^{\prime}\right)^{n}$ are set at the grid nodes.

New values of $X_{C}$ are obtained from the old ones subtracting the net outflow fluxes at the boundaries and adding the contributions from the source terms:

$$
\begin{aligned}
\rho_{C}^{n+1, *}= & \rho_{C}^{n}-\Delta t\left[\tilde{\mathbf{\nabla}} \cdot\left(P \mathbf{v} \theta^{-1}\right)\right]_{C}^{n+1 / 2, *} \\
(\rho \mathbf{v})_{C}^{n+1, *}= & (\rho \mathbf{v})_{C}^{n}-\Delta t\left[\tilde{\mathbf{v}} \cdot\left(P \mathbf{v} \circ \mathbf{v} \theta^{-1}+p^{n} \mathbf{I}\right)\right]_{C}^{n+1 / 2, *} \\
& -\Delta \operatorname{tg} \mathbf{k}\left[P / \theta+\left(\rho^{\prime}\right)^{n}\right]_{C}^{n+1 / 2, *} \\
P_{C}^{n+1, *}= & P_{C}^{n}-\Delta t[\tilde{\mathbf{v}} \cdot(P \mathbf{v})]_{C}^{n+1 / 2, *}
\end{aligned}
$$

where $\rho^{\prime}=(1-\alpha) \beta\left(\rho_{0} / \gamma p_{0}\right) p^{\prime}$. The superscripts $(\cdot)^{n+1 / 2, *}$ in (21) indicate effective time-averaged terms as they emerge from the chosen time integrator, and the asterisk indicates quantities evaluated in the course of the predictor step.
Note, we have rewritten the $\rho g$ term in the momentum equation in (21b) in terms of $P$ and $\theta$ using the equation of state [given by (2) for the FC model and (15) for the $\mathrm{PI}_{\rho, p}$ and $\mathrm{PI}_{\rho, p}^{\mathrm{tc}}$ models] where in the pseudoincompressible cases $P^{n+1 / 2, *} \equiv P_{0}$. In the compressible case, in agreement with second-order accuracy we use $P^{n+1 / 2, *}=P^{n}+(1 / 2) \delta p(\partial P / \partial p)$, where $\delta p$ here is the pressure increment computed in the correction step of the previous time loop. The derivative of $P$ with respect to $p$ is computed using the equation of state.

By writing $\rho g$ in this way we were able to decouple the buoyancy term from the small advective flux divergence errors that arise in the predictor step. Potential temperature effects can fully be accounted for in the predictor, because potential temperature is accurately advected and not affected by the divergence errors. However, the pressure does react to divergence errors. By relying on accurate pressure information computed during the previous time steps, the buoyancy term is shielded from this effect. As a result, this formulation was found to give models increased stability for larger time steps.

We have used the following symbolic notation to abbreviate the balance of a numerical flux, say $\mathbf{q}$, across gridcell boundaries:

$$
\tilde{\mathbf{\nabla}} \cdot \mathbf{q}_{C}=\frac{1}{|C|} \sum_{I \in \mathcal{I}_{C}} \mathbf{q}_{I} \cdot \mathbf{n}=\frac{1}{|C|} \oint_{\partial C} \mathbf{q} \cdot \mathbf{n} d \ell+O\left(\Delta x^{2}\right) .
$$

Here $\partial C$ is the boundary of cell $C$. See the appendix for further details on the numerical scheme used in the predictor.

Note that we discretize advection by considering $P \mathbf{v}$ as the carrier flux that transports (upwind) values of the advected quantities $(1 / \theta, \mathbf{v} / \theta, 1)$. This has turned out to be advantageous in many respects; for example, in the construction of a positivity preserving advection scheme in Klein (2009) [see also Smolarkiewicz et al. (2014) and references therein].

We consciously refrain from going into more detail here because many different combinations of secondorder accurate finite-volume space discretizations and time integrators can more or less interchangeably be employed for the predictor step, provided they are used in conjunction with a well-balanced discretization of the pressure-gradient and gravity terms (see, e.g., Botta et al. 2004; Klein 2009). The details of the scheme used to generate the results of section 4 are given in the appendix. At the end of the predictor step,

- the scalar variables $\rho, \theta$, and $P$ are second-order accurate (Klein 2009);

- the advecting fluxes $(P \mathbf{v})^{n+1 / 2}$ do not comply with the divergence constraint for $\alpha=0$, and they do not provide a stable update of $P$ for $\alpha>0$; and 
- using the old time level pressure in the momentum equation in (21b) prevents the scheme from being fully second-order accurate.

Crucially, for all values of $\alpha$ the time step used is limited by a CFL stability condition (Courant et al. 1928) independent of sound speed (see the appendix), so that we sidestep the stiffness induced by sound waves.

\section{b. Step 2: First correction}

The first correction step, which is the first of two linearly implicit substeps, corresponds to the Marker and Cell (MAC) projection in projection methods for incompressible flows (Bell et al. 1991). The advecting fluxes $P \mathbf{v}$ used in the predictor step do not abide by a semi-implicit discretization of the $P$ equation for the FC model and by the divergence constraint for the $\mathrm{PI}_{\rho, p}$ and $\mathrm{PI}_{\rho, p}^{\mathrm{tc}}$ models. In the first correction, an elliptic equation for a cell-centered pressure update $\delta p=p^{n+1}-p^{n}$ is derived by approximating (18c) at the half time level $t^{n+1 / 2}$, that is, by reconsidering

$$
\left[\alpha\left(\frac{\partial P}{\partial t}\right)+\nabla \cdot(P \mathbf{v})\right]^{n+1 / 2}=0 .
$$

The predictor step is discretized with second-order accuracy in time. As a consequence, the advecting fluxes $(P \mathbf{v})^{n+1 / 2, *}$ already include a first-order accurate update to the half time level according to the auxiliary equation system in (19), and this is sufficient to maintain secondorder accuracy for advection. Yet, for stability reasons an implicit correction is added that accounts for the influence of the new time level pressure gradient in the momentum equation in the following form (Klein 2009):

$$
(P \mathbf{v})^{n+1 / 2}=(P \mathbf{v})^{n+1 / 2, *}-\frac{\Delta t}{2} \theta^{n+1 / 2, *} \nabla \delta p .
$$

Again, the asterisk denotes predicted values. Since $\Delta t \delta p=$ $\Delta t\left(p^{n+1}-p^{n}\right)=O\left[(\Delta t)^{2}\right]$, this correction does not affect the second-order accuracy of advection. For $\alpha \neq 0$, the time derivative term is transformed as

$\left(\frac{\partial P}{\partial t}\right)^{n+1 / 2}=\left(\frac{\partial P}{\partial p} \frac{\partial p}{\partial t}\right)^{n+1 / 2}=\left(\frac{\partial P}{\partial p}\right)^{n+1 / 2, *} \frac{\delta p}{\Delta t}+O\left[(\Delta t)^{2}\right]$.

Using (24) and (25) in (23) we obtain the elliptic problem for any $\alpha \in[0,1]$,

$$
\begin{aligned}
& -\alpha\left(\frac{\mathcal{C}_{H}^{n+1 / 2, *}}{\Delta t} \delta p\right)_{C}+\tilde{\nabla} \cdot\left(\frac{\Delta t}{2} \theta^{n+1 / 2, *} \nabla \delta p\right)_{C} \\
& =\tilde{\nabla} \cdot\left[(P \mathbf{v})^{n+1 / 2, *}\right]_{C},
\end{aligned}
$$

where

$$
\mathcal{C}_{H}^{n+1 / 2, *}=\left(\frac{\partial P}{\partial p}\right)^{n+1 / 2, *} .
$$

The expression in (26) is responsible for determining stable time increments of $P$ in the compressible model $(\alpha=1)$, whereas it enforces the divergence constraint for $\alpha=0$.

With the solution of (26) $\delta p$ at hand, the advecting flux corrections read

$$
\delta P \mathbf{v} \cdot \mathbf{n}=-\frac{\Delta t}{2} \theta \nabla \delta p \cdot \mathbf{n},
$$

and the predicted values are corrected by

$$
\begin{aligned}
\rho_{C}^{n+1} & =\rho_{C}^{n+1, *}-\Delta t \tilde{\nabla} \cdot\left(\delta P \mathbf{v} \theta^{-1}\right)_{C}, \\
(\rho \mathbf{v})_{C}^{n+1, * *} & =(\rho \mathbf{v})_{C}^{n+1, *}-\Delta t \tilde{\nabla} \cdot\left(\delta P \mathbf{v} \circ \mathbf{v} \theta^{-1}\right)_{C}, \\
P_{C}^{n+1} & =P_{C}^{n+1, *}-\Delta t \tilde{\mathbf{\nabla}} \cdot(\delta P \mathbf{v})_{C} .
\end{aligned}
$$

where the advected variables $\theta^{-1}$ and $\mathbf{v} \theta^{-1}$ are evaluated at $(\cdot)^{n+1 / 2, *}$. The second asterisk indicates that the obtained value of the momentum is bound to receive a second correction as described below.

Note that (26) turns into a standard Poisson pressure projection equation for the pseudo-incompressible cases when $\alpha=0$. In these cases, the correction of $P$ in (29) automatically yields $P^{n+1} \equiv P_{0}$ up to the tolerance in the divergence term with which the Poisson equation was solved. Thus, in the pseudo-incompressible cases, the pressure variable $P$ is restored to its background value as a result of the first correction as it should be.

Thus far we have stabilized the advecting fluxes by incorporating an implicit pressure gradient contribution. We have not yet corrected the first-order error committed in the predictor step for the momentum equation by using the old time level pressure. This task is left to the second correction.

\section{c. Step 3: Second correction}

The use of the old time level pressure in the momentum equation in (21b) makes the predictor step first order accurate w.r.t. momentum. In a second correction step, the pressure and the momentum flux are corrected to achieve second-order accuracy and stability. Suppose we have already calculated an appropriate pressure update $\delta p=p^{n+1}-p^{n}$, then the correction of momentum reads

$$
(\rho \mathbf{v})_{C}^{n+1}=(\rho \mathbf{v})_{C}^{n+1, * *}-\frac{\Delta t}{2}\left[\tilde{\mathbf{\nabla}} \cdot(\delta p \mathbf{I})_{C}+\mathbf{k} \sigma \delta p\right],
$$


where

$$
\sigma=(1-\alpha) \beta \frac{g \rho_{0}}{\gamma p_{0}}
$$

Interpolating $\delta p$ as computed in the first correction from the cell centers to the cell interfaces and using these data to evaluate (30) turns out to generate an unstable update. We avoid this by solving a second elliptic problem for a node-centered pressure variable (see similar procedures in Almgren et al. 1998; Schneider et al. 1999; Klein 2009; Vater and Klein 2009). To derive the second elliptic equation, we multiply (30) by $\theta^{n+1}$ taking into account that the scalars $\rho, P$, and $\theta$ have already attained their final values after the first correction and are unchanged in the second one. This yields

$$
(P \mathbf{v})_{C}^{n+1}=(P \mathbf{v})_{C}^{n+1, * *}-\frac{\Delta t}{2} \theta_{C}^{n+1}\left[\tilde{\mathbf{v}} \cdot(\delta p \mathbf{l})_{C}+\mathbf{k} \sigma \delta p\right] .
$$

As in the first correction we insert (32) into

$$
\alpha\left(\frac{\partial P}{\partial t}\right)^{n+1 / 2}+\nabla \cdot\left[\frac{2-\alpha}{2}(P \mathbf{v})^{n+1}+\frac{\alpha}{2}(P \mathbf{v})^{n}\right]=0,
$$

where, for $\alpha=1$, a second-order accurate midpoint discretization with no off-centering is considered. After node-centered space discretization of the divergence, we obtain the elliptic problem:

$$
\begin{aligned}
- & \alpha\left(\frac{\mathcal{C}_{H}^{n+1}}{\Delta t} \delta p\right)_{\bar{C}}+\tilde{\mathbf{\nabla}} \cdot\left[\frac{(2-\alpha) \Delta t}{4} \theta^{n+1}(\nabla \delta p+\mathbf{k} \sigma \delta p)\right]_{\bar{C}} \\
& =\tilde{\mathbf{\nabla}} \cdot\left[\frac{2-\alpha}{2}(P \mathbf{v})^{n+1, * *}+\frac{\alpha}{2}(P \mathbf{v})^{n}\right]_{\bar{C}}
\end{aligned}
$$

where $\mathcal{C}_{H}^{n+1}$ is defined by (27) using the corrected value of $P$.

As in the first correction, we obtain a Helmholtz equation for $\alpha=1$ where the zero-order term accounts for compressibility. The difference between FC $(\alpha=1)$ and $\mathrm{PI}_{\rho, p}^{\mathrm{tc}}(\alpha=0)$ is a modified structure of the system matrix.

We note that in the fully compressible case a backward difference (BDF2) discretization can be used, as done in Vater (2013). In that case, and for $\alpha=1$, (34) is replaced with

$$
\begin{gathered}
-\left(\frac{3 \mathcal{C}_{H}^{n+1}}{2 \Delta t} \delta p\right)_{\bar{C}}+\frac{2}{3} \Delta t \tilde{\nabla} \cdot\left(\theta^{n+1} \tilde{\mathbf{\nabla}} \delta p\right)_{\bar{C}} \\
=\tilde{\mathbf{\nabla}} \cdot(P \mathbf{v}) \bar{C}_{\bar{C}}^{n+1}-\left(\frac{\mathcal{C}_{H}^{n+1}}{2 \Delta t} \delta p^{\text {old }}\right)_{\bar{C}},
\end{gathered}
$$

where $\delta p^{\text {old }}=p^{n}-p^{n-1}$ denotes the old time level pressure increment.

A nine-point stencil is used for the discretization of the Laplacian equation in (34) or (35), which is obtained as follows: the nodal values define continuous piecewise bilinear pressure distributions on the primary control volumes. We integrate their gradients analytically over the boundaries of the dual cells that are centered on the grid nodes. The solution $\delta p$ is accordingly defined in the centers of the dual cells, $\bar{C}$. Straightforward numerical integration of pressures over the primary cell interfaces can thus be employed in evaluating the second momentum correction in (30). After the nodal pressures have been updated to the new time level as well, all variables are now second-order accurate and ready for the next time step. See details of the discretization in the appendix.

\section{Numerical results}

In this section, we present the results of the simulations performed with our semi-implicit method. The aim is to show that the model numerics produce results in agreement with its theoretical properties in different configurations. First, a convergence study in the FC configuration is presented. Then, results with FC and pseudo-incompressible $\left(\mathrm{PI}_{\rho, p}\right)$ models are compared on simulations of thermal perturbations. The impact of the thermodynamic consistency $\left(\mathrm{PI}_{\rho, p}^{\mathrm{tc}}\right)$ term is also evaluated.

The numerical model is implemented in an object oriented $\mathrm{C}++$ environment based on the SAMRAI framework for mesh refinement (Hornung et al. 2006). Krylov-type methods with algebraic multigrid preconditioners as included in the Hypre library (Falgout et al. 2006) are used to solve the linear systems in the correction step. Our coding framework is fully parallelized and 3D ready. However, an extensive analysis of its parallel efficiency lies outside the scope of the present work.

\section{a. Convergence study}

First, we assess the accuracy properties of the FC model on a case of pure transport in a highly idealized setting with $g=0$. The case (Kadioglu et al. 2008) consists of a traveling rotating vortex in the doubly periodic 
unit-square-shaped domain $\Omega=[0,1]^{2} \mathrm{~m}^{2}$. The vortex is axisymmetric and rotates counterclockwise with unitary velocity. Density is modeled by a smooth, nonconstant function and a constant and unitary transport velocity $\mathbf{v}=(1,1)^{\mathrm{T}} \mathrm{m} \mathrm{s}^{-1}$ is superimposed. The vortex is an exact solution for the zero Mach number incompressible equations, to which $\mathrm{PI}_{\rho, p}^{\mathrm{tc}}$ and $\mathrm{PI}_{\rho, p}$ reduce in the absence of gravity (Klein 2009). With the pressure field correctly initialized, it is an exact solution for the fully compressible equations as well. We refer to Kadioglu et al. (2008) for the initial data not reported here for brevity. Note that some of the coefficients in the expression for initial pressure were incorrectly reported in Kadioglu et al. (2008), the correct expression is available upon request.

In the compressible case, the initial distribution for $P$ is derived via the equation of state in (3). Reference physical quantities are set as follows:

$\rho_{\text {ref }}=0.5 \mathrm{~kg} \mathrm{~m}^{-3}, \quad p_{\text {ref }}=101625 \mathrm{~Pa}, \quad T_{\text {ref }}=706.098 \mathrm{~K}$,

corresponding to a maximum Mach number $M_{\max }=$ $\max \left(\|\mathbf{v}\|_{\mathrm{RMS}} \sqrt{\gamma p / \rho}\right)=4.96 \times 10^{-3}$. The high value of $T_{\text {ref }}$ is computed from $p_{\text {ref }}$ and $\rho_{\text {ref }}$ considered in Kadioglu et al. (2008) and enables an easier comparison with their results for the density.

The flow is simulated by running the FC semi-implicit model $(\alpha \equiv 1)$ on a grid with 192 cells in both directions at $\mathrm{CFL}=0.45$, that is, constant $\Delta t=\Delta t_{A}=9.7 \times 10^{-4} \mathrm{~s}$ and $\Delta x=5.21 \times 10^{-3} \mathrm{~m}$. These data correspond to a sound-speed-based $\mathrm{CFL}_{S}=\mathrm{CFL} / M_{\max } \approx 90.72$.

The vortex is transported by the background unitary velocity. Because of the doubly periodic boundary, the initial configuration is reproduced unchanged at time $T=1$ s (Fig. 2). Similar results (not shown) are obtained for momentum and $P$ in FC runs and for all variables except for $P$ (which is constant) in $\mathrm{PI}_{\rho, p}^{\mathrm{tc}}$ runs.

Furthermore, the numerical solution converges quadratically in the maximum norm (Fig. 3). The experimental order of accuracy is in agreement with the theoretical accuracy of the scheme presented in section 3. Similar results are obtained with $\mathrm{PI}_{\rho, p}^{\mathrm{tc}}$ runs (not shown).

The FC results shown above validate the use of the fully compressible flow solver that extends the pseudoincompressible framework of Klein (2009).
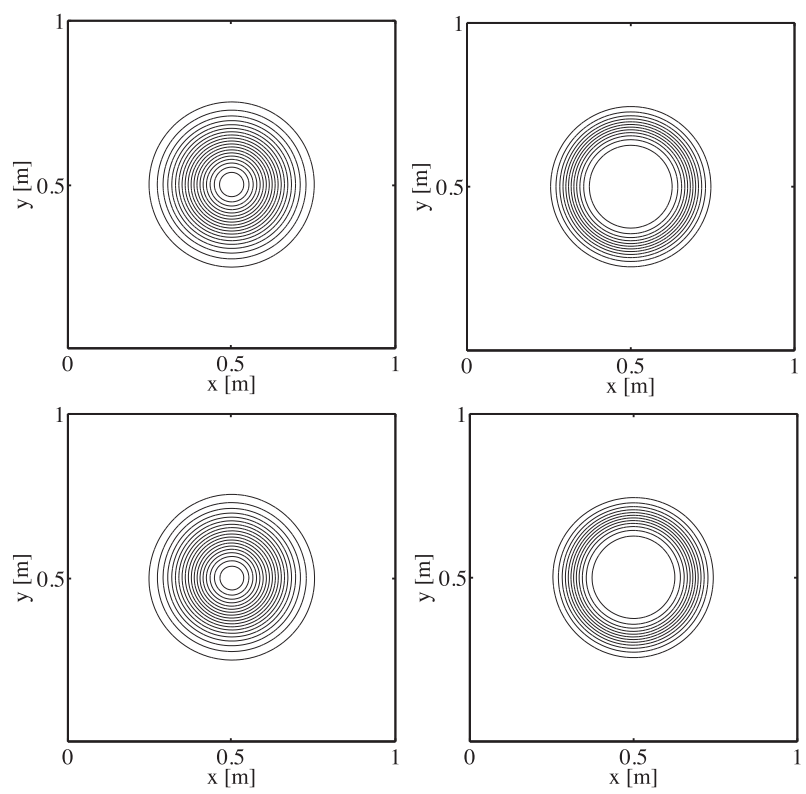

FIG. 2. Smoothed rotating vortex results: (left) density and (right) pressure. (top) Initial data and (bottom) computed values at $T=1 \mathrm{~s}$ with the FC model. Contours are plotted every $0.025 \mathrm{~kg} \mathrm{~m}^{-3}$ (from 0.525 to $0.975 \mathrm{~kg} \mathrm{~m}^{-3}$ ) for density and every $0.025 \mathrm{~Pa}$ (from -0.025 to $-0.3 \mathrm{~Pa}$ ) for pressure. The domain is discretized with 192 cells in each direction with $\mathrm{CFL}=0.45$.

\section{b. Rising bubble}

Next, we consider a warm air bubble test case in the domain $\Omega=(x, z) \in[-10,10] \times[0,10] \mathrm{km}^{2}$. We set the following initial data for a homentropic atmosphere (Botta et al. 2004):

$$
\begin{aligned}
& p(z)=p_{\text {ref }}\left(1-\Gamma \frac{g \rho_{\text {ref }}}{p_{\text {ref }}} z\right)^{1 / \Gamma}, \quad \rho(z)=\rho_{\text {ref }}\left[\frac{p(z)}{p_{\text {ref }}}\right]^{1 / \gamma}, \\
& \rho_{\text {ref }}=\frac{p_{\text {ref }}}{R T_{\text {ref }}}
\end{aligned}
$$

where, in agreement with Klein (2009), $\rho_{\text {ref }}, p_{\text {ref }}, g$, and $T_{\text {ref }}$ have the values $1 \mathrm{~kg} \mathrm{~m}^{-3}, 8.61 \times 10^{4} \mathrm{Nm}^{-2}$, $10 \mathrm{~m} \mathrm{~s}^{-2}$, and $300 \mathrm{~K}$, respectively, and $\Gamma=(\gamma-1) / \gamma$. The background potential temperature $\theta$ is constant. The homentropic setting in (37) is perturbed with a smoothed cone-shaped thermal perturbation $\theta^{\prime}$, given by Klein 2009 :

$$
\theta^{\prime}(x, z)=\left\{\begin{array}{ll}
\delta \theta \cos ^{2}\left(\frac{\pi}{2} r\right) & (r \leq 1) \\
0 & \text { otherwise }
\end{array},\left\{\begin{array}{l}
\delta \theta=2 \mathrm{~K} \\
r=5 \sqrt{\left(\frac{x}{L}\right)^{2}+\left(\frac{z}{L}-\frac{1}{5}\right)^{2}} \\
L=10 \mathrm{~km}
\end{array}\right.\right.
$$



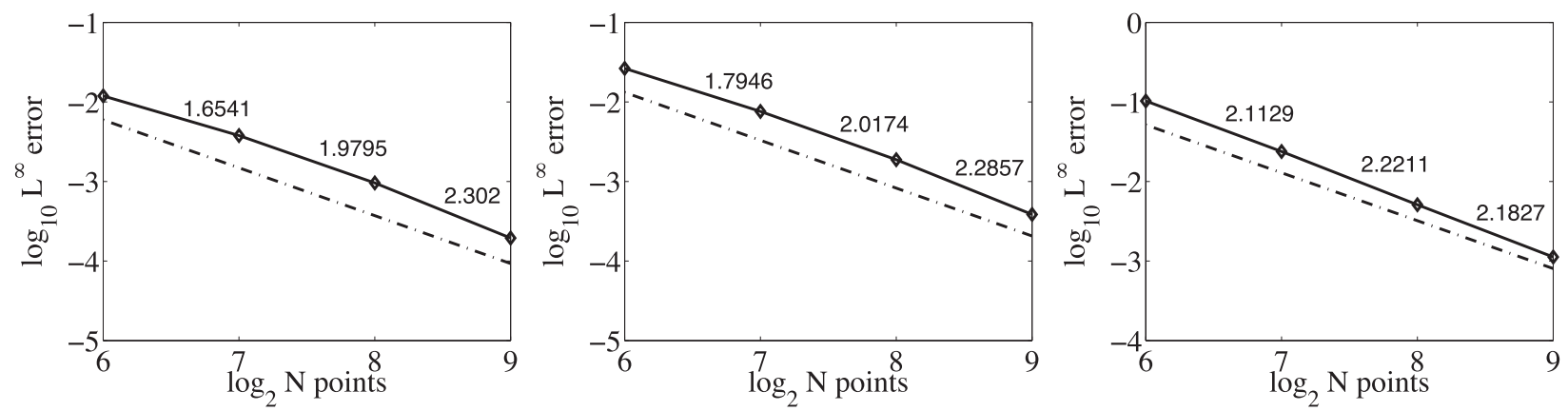

FIG. 3. Smoothed rotating vortex results: (left) density, (middle) momentum norm, and (right) pressure convergence plots. Errors are shown in the maximum norm of computed solutions at $T=1 \mathrm{~s}$ on grids with $64^{2}, 128^{2}, 256^{2}$, and $512^{2}$ cells with respect to computed solutions on a reference grid with $1024^{2}$ cells. The numbers inside the graphs are the experimental rates of convergence between subsequent grid refinements. The dashed-dotted line represents the quadratic slope.

The initial velocity is zero. Lateral boundary conditions are periodic, with solid walls on top and bottom boundaries.

We run our semi-implicit trapezoidal scheme on a grid with $\Delta x=\Delta z=125 \mathrm{~m}$ (i.e., $160 \times 80$ cells) and CFL $=$ 0.5 . In the first five steps a buoyancy-driven time step $\left(\Delta t=\Delta t_{B} \approx 21.69 \mathrm{~s}\right)$ is used. Because of growing velocities, the advection-driven time step is used for the remainder of the simulation. Toward the end of the simulation, values of $\Delta t \approx 4.6 \mathrm{~s}$ are attained.

Driven by buoyancy, the warm bubble rises and rolls up on the sides (Fig. 4). The amplitude of the thermal perturbation at final time $T=1000 \mathrm{~s}$ is in agreement with the results in Klein (2009), as shown in Table 2.
However, the $\mathrm{PI}_{\rho, p}$ bubble rises faster, is not as wide, and exhibits a phase shift with respect to both the $\mathrm{PI}_{\rho, p}^{\mathrm{tc}}$ and the FC models (Fig. 5).

The discrepancies in the $\mathrm{PI}_{\rho, p}$ model come from neglecting the effect of pressure perturbations on the buoyancy. The extra buoyancy term present in the $\mathrm{PI}_{\rho, p}^{\mathrm{tc}}$ model reduces buoyancy near the top of the bubble because of an increase in pressure near the bubble top and increases buoyancy at the two tails due to a pressure decrease near the tails. Furthermore, the overall buoyancy of the bubble decreases causing a decrease in the phase speed. Therefore, the $\mathrm{PI}_{\rho, p}^{\mathrm{tc}}$ bubble is both lower and wider than the $\mathrm{PI}_{\rho, p}$ model and, as a result, resembles the FC model more closely.
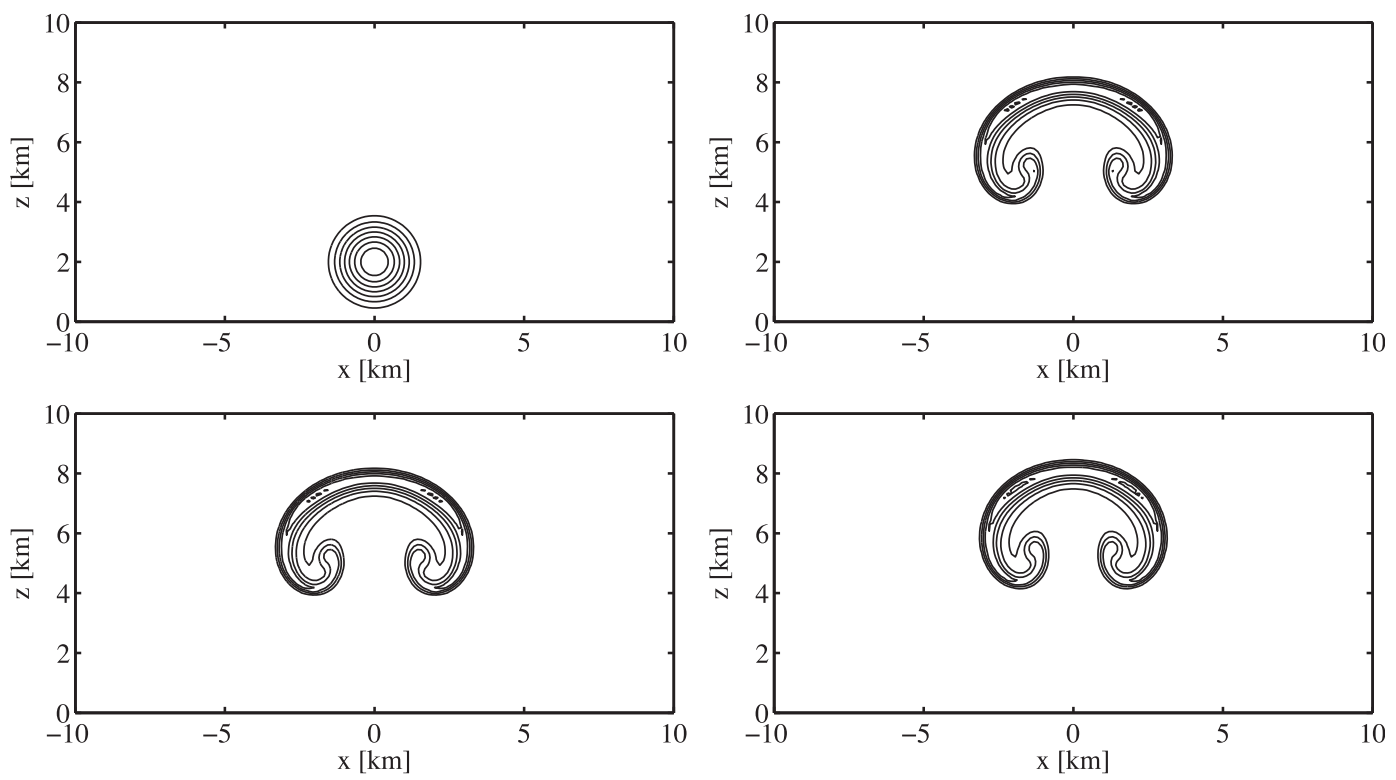

FIG. 4. Rising bubble results. Potential temperature (top left) initial data and computed value at $T=1000 \mathrm{~s}$ with the (top right) FC, (bottom left) $\mathrm{PI}_{\rho, p}^{\mathrm{tc}}$, and (bottom right) $\mathrm{PI}_{\rho, p}$ models. Contours are plotted every $0.25 \mathrm{~K}$ starting at $300.25 \mathrm{~K}$. 
TABLE 2. Rising bubble results: maximum temperature perturbation $\theta_{\max }^{\prime}$, attained height $z_{\max }$, and horizontal extension $x_{\max }-x_{\min }$ at final time $T=1000 \mathrm{~s}$ for $\mathrm{FC}, \mathrm{PI}_{\rho, p}^{\text {tc }}$, and $\mathrm{PI}_{\rho, p}$ models. The values refer to the external contour $\theta^{\prime}=0.25 \mathrm{~K}$.

\begin{tabular}{lccc}
\hline \hline & $\theta_{\max }^{\prime}(\mathrm{K})$ & $z_{\max }(\mathrm{m})$ & $x_{\max }-x_{\min }(\mathrm{m})$ \\
\hline $\mathrm{FC}$ & 1.64 & 8183 & 6637 \\
$\mathrm{PI}_{\rho, p}^{\mathrm{tc}}$ & 1.64 & 8187 & 6648 \\
$\mathrm{PI}_{\rho, p}$ & 1.65 & 8469 & 6278 \\
\hline
\end{tabular}

Results with $\mathrm{PI}_{\rho, p}^{\mathrm{tc}}$ as measured in a one-dimensional cut of $\theta^{\prime}$ at height $z=7500$ match the FC results within a $2 \%$ error (Table 3 ).

Results with the PI $\mathrm{I}_{\rho, p}^{\mathrm{tc}}$ model do not differ substantially from FC results at the end of the simulation at $T=$ $1000 \mathrm{~s}$. The different dynamics of the FC case can be detected in the onset of sound waves in the initial stages of the simulation. With the FC model $(\alpha=1)$ the initial potential temperature perturbation triggers acoustic waves. These are visible in the top-left panel of Fig. 6, which displays pressure increments at time $t=26.6 \mathrm{~s}$ in a run of the FC model with $\Delta t=\Delta t_{I}=1.9 \mathrm{~s}$. The oscillations are due to the initial hydrostatic pressure distribution from (37) not being acoustically balanced.

The presence of associated pressure oscillations is confirmed by a time series over the first $350 \mathrm{~s}$ of the pressure time increment values recorded at the point $(x, z)=(-7.5,5) \mathrm{km}$ marked with a cross in the top-left panel of Fig. 6 . The time series are shown in the topright, bottom-left, and bottom-right panels of Fig. 6. The top-right and bottom-left plots are relative to simulations at constant $\Delta t=\Delta t_{\mathrm{I}}=1.9 \mathrm{~s}$. The simulation relative to the bottom-right panel is at CFL $\approx 0.5$ as in Fig. 4 .

The FC model results (solid lines in all plots) display oscillations triggered by the initial pressure imbalance. The amplitude of the acoustic oscillations in the small time step case (top-right panel) is ninefold the amplitude of the large time step runs (bottom-right panel). The effect is suppressed in the $\mathrm{PI}_{\rho, p}$ runs (dashed lines) except for an initial transient. Note that in the large time step run, the initial transient masks the amplitude of the acoustics. Therefore, the data of the first time step was removed in the bottom-right panel of Fig. 6 .

In the case of the $\mathrm{PI}_{\rho, p}$ model, pressure is determined by the solution of a time-independent Poisson problem, which describes the pressure field in the absence of sound waves. The quantity $\mathrm{PI}_{\rho, p}$ is considered because the extra $\mathrm{PI}_{\rho, p}^{\mathrm{tc}}$ term does not modify the results as far as acoustics are concerned. On the one hand, the reduction in the amplitude of the large time step acoustic oscillations shows that the semi-implicit method is able to handle acoustic oscillations at CFL numbers independent of the sound speed. On the other hand, the effect of acoustics is not completely suppressed in the large time step, either.

However, thanks to the blending feature, the code is able to continuously transition from the $\mathrm{PI}_{\rho, p}$ configuration to the FC configuration. The bottom-left panel of Fig. 6 shows the time series of pressure increments for blended runs. We set the transition parameter $\alpha$ from section 2 to zero for $S_{1}$ time steps. Then, $\alpha$ increases linearly to $\alpha=1$ over $S_{2}$ time steps. Starting at the time step number $S_{1}+S_{2}$, the code runs compressibly with $\alpha=1$.

In the bottom-left panel of Fig. 6, the thin solid line in the background denotes the fully compressible run. The dashed-dotted curve and thick solid curves were obtained with $S_{2}=20$ and $S_{2}=40$, respectively. There are no disturbances for the first $S_{1}=10$ pseudoincompressible steps in these two pressure graphs, and the results coincide with those from the run of the $\mathrm{PI}_{\rho, p}$ model (dashed line in the right panels). Perturbations
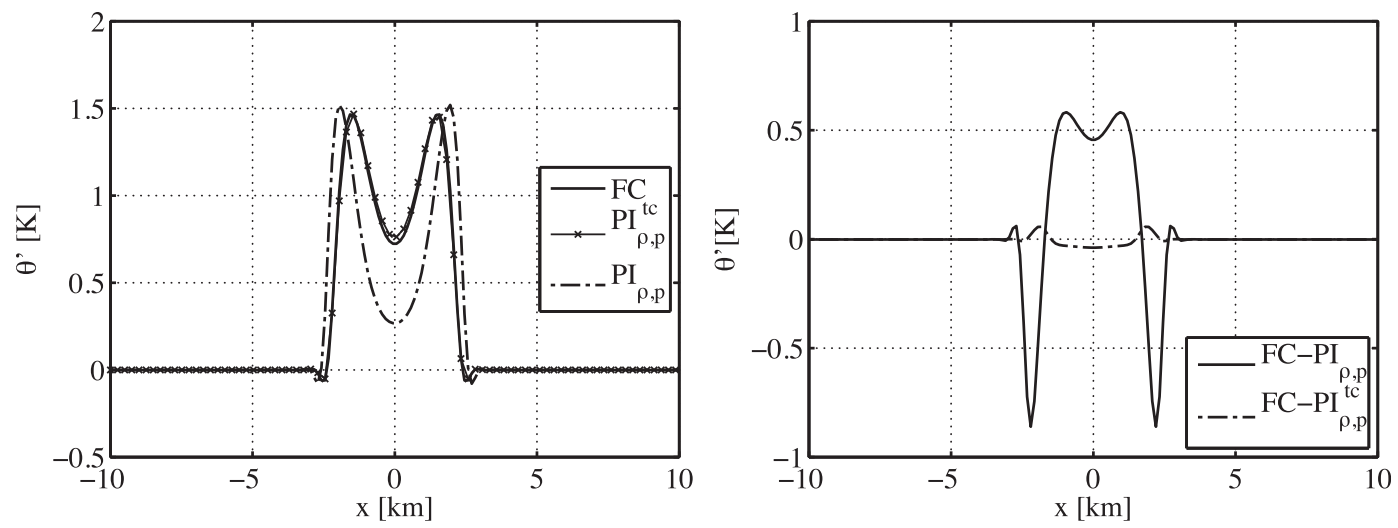

FIG. 5. Rising bubble results: potential temperature perturbation at final time $T=1000 \mathrm{~s}$. (left) A horizontal cut of the final $\theta^{\prime}$ at height $z=7500 \mathrm{~m}$ for the FC (solid line), $\mathrm{PI}_{\rho, p}^{\mathrm{tc}}$ (cross-marked line), and $\mathrm{PI}_{\rho, p}$ (dashed-dotted line). (right) The difference from the FC cut of the $\mathrm{PI}_{\rho, p}^{\mathrm{tc}}$ cut (dashed-dotted line) and the $\mathrm{PI}_{\rho, p}$ cut (solid line). 
TABLE 3. Rising bubble results: relative root-mean-square error $E_{\text {rel }}^{\mathrm{rms}}$ and maximum error $E_{\mathrm{rel}}^{\mathrm{max}}$ on potential temperature perturbation profile $\theta^{\prime}$ and maximum error $E_{\mathrm{rel}}^{\max }$ on the maximum perturbation amplitude $\theta_{\max }^{\prime}$ for the $\mathrm{PI}_{\rho, p}^{\mathrm{tc}}$ and $\mathrm{PI}_{\rho, p}$ cuts at $z=7500 \mathrm{~m}$ with respect to the FC cut as in Fig. 5.

\begin{tabular}{lccc}
\hline \hline & $E_{\mathrm{rel}}^{\mathrm{rms}}\left(\theta^{\prime}\right)$ & $E_{\mathrm{rel}}^{\max }\left(\theta^{\prime}\right)$ & $E_{\mathrm{rel}}^{\max }\left(\theta_{\text {max }}^{\prime}\right)$ \\
\hline $\mathrm{PI}_{\rho, p}^{\mathrm{tc}}-\mathrm{FC}$ & 0.017 & 0.018 & $1.07 \times 10^{-3}$ \\
$\mathrm{PI}_{\rho, p}-\mathrm{FC}$ & 0.57 & 0.57 & $3.61 \times 10^{-2}$ \\
\hline
\end{tabular}

arise in the transitional period and fully develop after $S_{1}+S_{2}$ time steps. The oscillations' amplitudes in the blended runs are considerably lower than those of the FC run and they are lower for the larger $S_{2}$ value (i.e., the longer transitional period).

Results in the bottom-left panel of Fig. 6 demonstrate the capabilities of the blended model. Acoustic perturbations are absent when the model runs in pseudo-incompressible mode with $\alpha=0$ and they emerge significantly damped after the transition to $\alpha=1$ in fully compressible mode. Therefore, when blended continuously with the compressible discretization, the soundproof limit discretization can be used to actively control imbalances in the initial data. The oscillation amplitudes are substantially reduced also when larger time steps are employed as seen in the bottom-right panel of Fig. 6.

Finally, as in Almgren et al. (2006a), which presents a pseudo-incompressible code for stellar hydrodynamics, we compare plots of the Mach number in the initial stages of $\mathrm{FC}, \mathrm{PI}_{\rho, p}$, and blended runs. Results at time $t=21.66 \mathrm{~s}$, that is, time step number 57 at $\Delta t=\Delta t_{\mathrm{I}}=$ $0.38 \mathrm{~s}$, are displayed in Fig. 7. The mushroom-shaped FC result (left panel) reveals the initial onset of sound waves due to pressure imbalances already inspected in Fig. 6, while the $\mathrm{PI}_{\rho, p}$ plot (middle panel) and blended plot (right panel) show no perturbation away from the
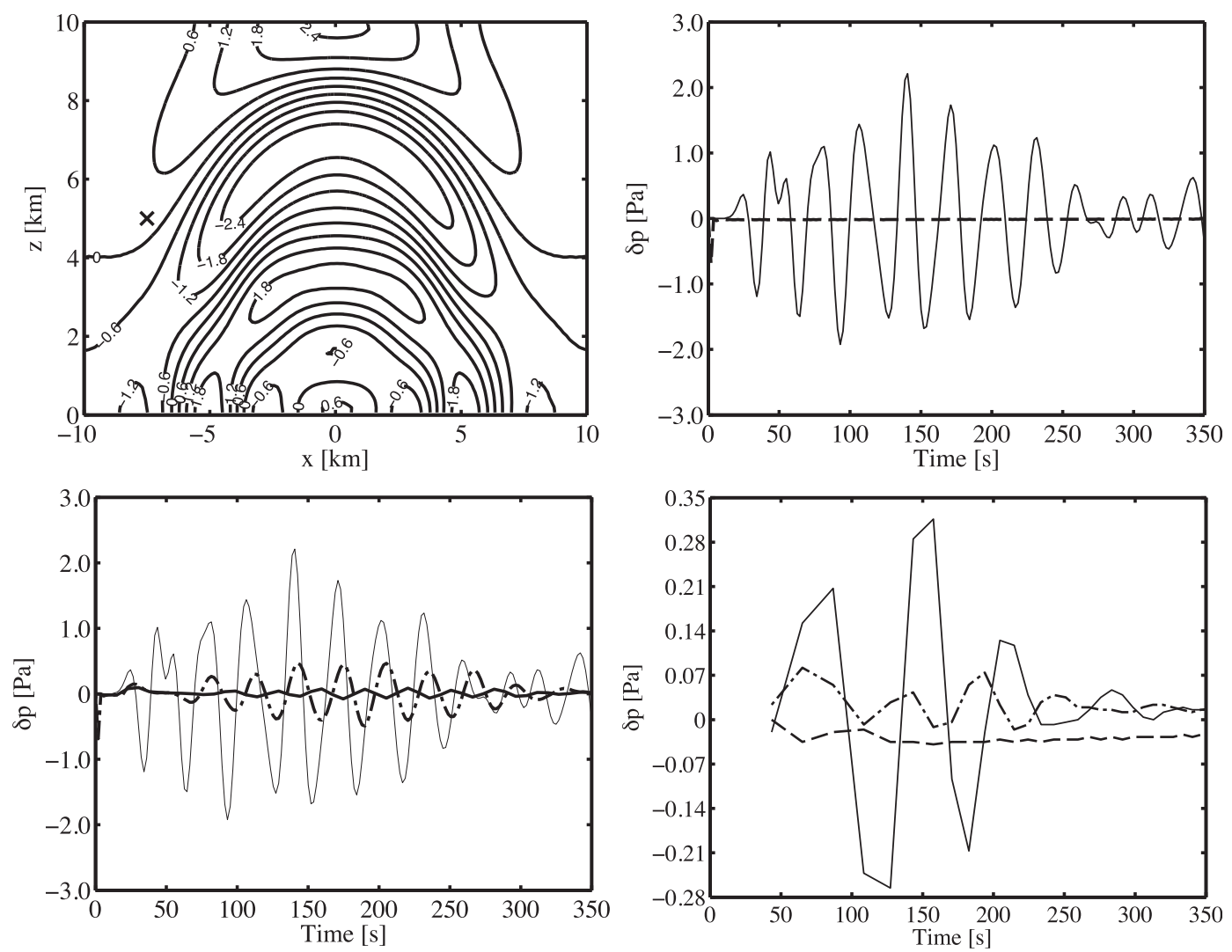

FIG. 6. Rising bubble results, nodal pressure time increment $\delta p$. (top left) Contours of $\delta p$ every 0.6 Pa starting at $-3 \mathrm{~Pa}$, time step $14(t=26.6 \mathrm{~s})$ for the FC model. (top right) History of $\delta p$ over the first $350 \mathrm{~s}$ measured at $(x, z)=$ $(-7.5,5) \mathrm{km}$ for FC (solid line) and $\mathrm{PI}_{\rho, p}$ (dashed line) configurations; constant time step $\Delta t=1.9 \mathrm{~s}$. (bottom left) History of $\delta p$ over the first $350 \mathrm{~s}$ measured at the same location. Blended runs at constant $\Delta t=1.9 \mathrm{~s}$ with $S_{1}=10$ initial pseudo-incompressible steps and $S_{2}=20$ (dashed-dotted line) and $S_{2}=40$ (thick solid line) transition steps are compared with the fully compressible run, $S_{1}=S_{2}=0$ (thin solid line). (bottom right) As in (top right), the dasheddotted line refers to a blended run with $S_{1}=0, S_{2}=3$; the time step is determined by CFL $=0.5$ (initial $\Delta t \approx 21.69 \mathrm{~s}$ ); and the data for the first time step are removed. 

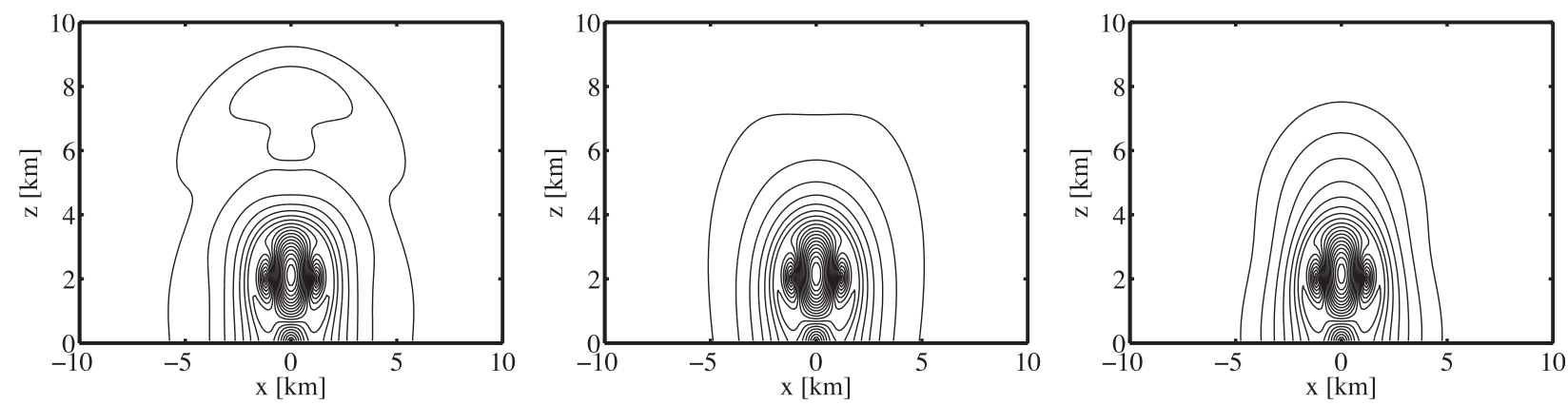

FIG. 7. Rising bubble results: Mach number $M$ at time step $56(T \approx 21.66 \mathrm{~s}$ for $\Delta t=0.38 \mathrm{~s})$. (left) FC model, $S_{1}=S_{2}=0 ;($ middle $)$ PI $\rho, p$ model; and (right) $\mathrm{PI}_{\rho, p}$ then FC model, $S_{1}=10, S_{2}=40$. Contours are plotted every $10^{-4}$ in the range [0.0001, 0.002].

bubble. A very small time step was considered in this case following Almgren et al. (2006a) in order to track more closely the dynamics in the initial stages.

\section{c. Density current}

This test (Straka et al. 1993) consists of a negative potential temperature perturbation in a $[-25.6,25.6] \times$ $[0,6.4] \mathrm{km}^{2}$ homentropic atmosphere in $(37)$,

$$
T^{\prime}=\left\{\begin{array}{lll}
0 \mathrm{~K} & \text { if } \quad r>1 \\
-15[1+\cos (\pi r)] / 2 \mathrm{~K} & \text { if } \quad r<1
\end{array},\right.
$$

where $r=\left\{\left[\left(x-x_{c}\right) / x_{r}\right]^{2}+\left[\left(z-z_{c}\right) / z_{r}\right]^{2}\right\}^{0.5}, x_{c}=0 \mathrm{~km}$, $x_{r}=4 \mathrm{~km}, z_{c}=3 \mathrm{~km}$, and $z_{r}=2 \mathrm{~km}$. From $\theta=T\left(p / p_{\text {ref }}\right)^{-\Gamma}$ we derive the potential temperature perturbation and density distribution:

$$
\theta^{\prime}(x, z)=\frac{T^{\prime}}{1-\Gamma \frac{g \rho_{\text {ref }}}{p_{\text {ref }}} z}, \quad \rho(z)=\rho_{\text {ref }}\left[\frac{p(z)}{p_{\text {ref }}}\right]^{1 / \gamma} \frac{\theta_{\text {ref }}}{\theta_{\text {ref }}+\theta^{\prime}},
$$

where $\theta_{\text {ref }}=T_{\text {ref. }}$. The boundary conditions are periodic on the left and right boundary, with solid walls on the top and bottom boundary. Furthermore, we add an artificial diffusion term $\rho \mu \nabla^{2} \mathbf{v}$ to the right-hand side of the momentum equation $\left(\rho \mu \nabla^{2} \theta\right.$ in the $P$ equation), with $\mu=75 \mathrm{~m}^{2} \mathrm{~s}^{-1}$ as in Straka et al. (1993). The initial velocity is set to zero, and the reference quantities are $T_{\text {ref }}=$ $300 \mathrm{~K}, p_{\text {ref }}=10^{5} \mathrm{~Pa}$, and $\rho_{\text {ref }}=p_{\text {ref }} /\left(R T_{\text {ref }}\right)$.

The models are run with $\Delta x=50 \mathrm{~m}$ and CFL $=0.5$. Thus, the time step is $\Delta t=\Delta t_{B} \approx 4.65 \mathrm{~s}$ for the first three steps and then the advective time step is used. For the FC model, a backward difference approach in the second projection is used, see (35). Because of the symmetrical nature of the test case, only the plots for the subdomain $[0,19.2] \times[0,4.8] \mathrm{km}^{2}$ are shown.

Obtained values of the final thermal perturbation and the front positions as calculated by the $\mathrm{FC}$ and $\mathrm{PI}_{\rho, p}^{\mathrm{tc}}$ models (Fig. 8 and Table 4) are in line with results in the literature (Straka et al. 1993; Restelli and Giraldo 2009). In contrast to the rising bubble case, the extra buoyancy term in the $\mathrm{PI}_{\rho, p}^{\mathrm{tc}}$ model results in an overall increase in the buoyancy of the bubble. This increase in buoyancy causes the bubble to fall slower and reduces the phase speed when compared with the $\mathrm{PI}_{\rho, p}$ model. This can be seen in the farther front position and in the horizontal cut at height $z=1200 \mathrm{~m}$ (Fig. 9) of the $\mathrm{PI}_{\rho, p}$ model when compared to both the FC and
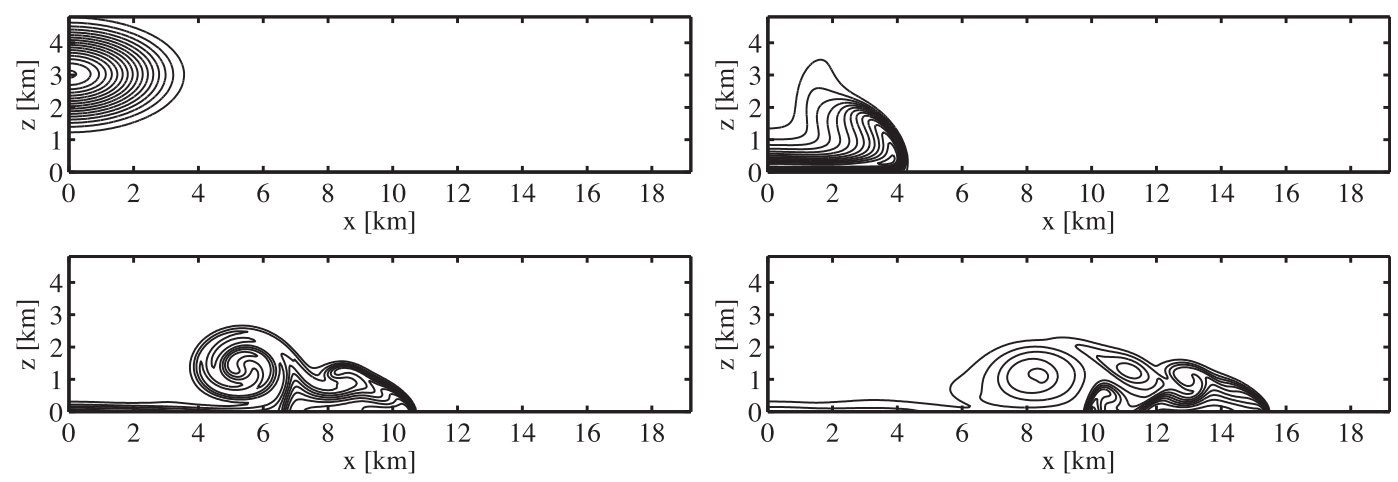

FIG. 8. Density current results: potential temperature perturbation. (top left) Initial data, FC results at (top right) $t=300 \mathrm{~s}$, (bottom left) $t=600 \mathrm{~s}$, and (bottom left) $t=900 \mathrm{~s}$. Contours are plotted every $1 \mathrm{~K}$ from -16.5 to $-0.5 \mathrm{~K}$. 
TABLE 4. Density current results: maximum temperature perturbation $\theta_{\max }^{\prime}$ and front position $x_{\max }$ at final time $T=900 \mathrm{~s}$. The quantity $x_{\max }$ is the rightmost intersection of the $1-\mathrm{K}$ contour with the bottom boundary.

\begin{tabular}{lcc}
\hline \hline & $\theta_{\max }^{\prime}(\mathrm{K})$ & $x_{\max }(\mathrm{m})$ \\
\hline $\mathrm{FC}$ & -10.14 & 15476 \\
$\mathrm{PI}_{\rho, p}^{\mathrm{tc}}$ & -10.17 & 15456 \\
$\mathrm{PI}_{\rho, p}$ & -9.96 & 15676 \\
\hline
\end{tabular}

$\mathrm{PI}_{\rho, p}^{\mathrm{tc}}$ models. As a result, the $\mathrm{PI}_{\rho, p}$ model displays considerable deviations (higher than $40 \%$ ) relative to $\mathrm{FC}$ runs (Table 5). For the $\mathrm{PI}_{\rho, p}^{\mathrm{tc}}$ model, the deviation from $\mathrm{FC}$ is lower than $5 \%$.

\section{d. Inertia-gravity waves}

Next, we consider a thermally stratified atmosphere with stable stratification of potential temperature $\partial \theta / \partial z>0$. In particular, as in Restelli and Giraldo (2009) and Skamarock and Klemp (1994), we take

$$
\theta(z)=T_{\text {ref }} \exp \left(\frac{N^{2}}{g} z\right)
$$

where $N$ denotes the buoyancy frequency. With $N=$ $0.01 \mathrm{~s}^{-1}, g=9.81 \mathrm{~m} \mathrm{~s}^{-2}$, and $T_{\text {ref }}=300 \mathrm{~K}$, we have $\theta \in$ $[300,332.19] \mathrm{K}$ for $z \in[0,10] \mathrm{km}$. The other variables are defined as

$$
\begin{aligned}
& p(z)=p_{\text {ref }}\left\{1-\frac{g}{N^{2}} \Gamma \frac{g \rho_{\text {ref }}}{p_{\text {ref }}}\left[1-\exp \left(-\frac{N^{2} z}{g}\right)\right]\right\}^{1 / \Gamma}, \\
& \rho(z)=\rho_{\text {ref }}\left(\frac{p(z)}{p_{\text {ref }}}\right)^{1 / \gamma} \exp \left(-\frac{N^{2} z}{g}\right), \quad \rho_{\text {ref }}=\frac{p_{\text {ref }}}{R T_{\text {ref }}},
\end{aligned}
$$

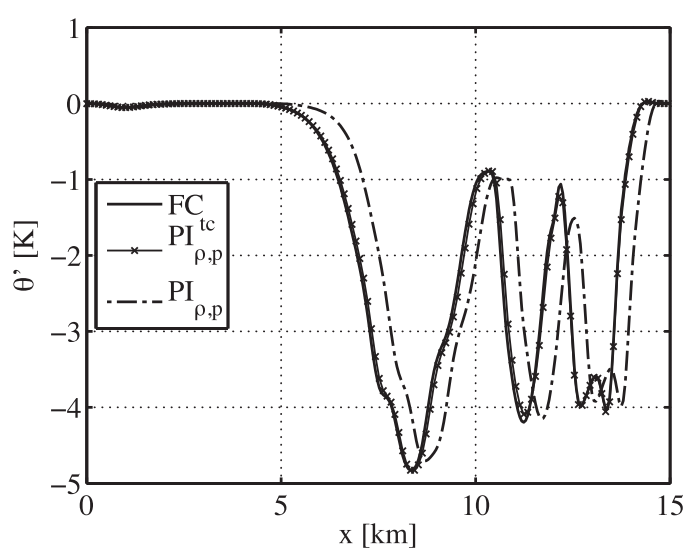

with $p_{\text {ref }}=10^{5} \mathrm{~Pa}$. On top of the background stratification in $(41)-(42)$, in a $[0,300] \times[0,10] \mathrm{km}^{2}$ domain we consider the perturbation [Skamarock and Klemp (1994) and Fig. 10 left panel]:

$$
\theta^{\prime}(x, z, 0)=0.01 \mathrm{~K} \frac{\sin (\pi z / H)}{1+\left[\left(x-x_{c}\right) / a\right]^{2}},
$$

with $H=10 \mathrm{~km}, x_{c}=100 \mathrm{~km}$, and $a=5 \mathrm{~km}$. In addition, there is a background horizontal flow $u=20 \mathrm{~m} \mathrm{~s}^{-1}$. The simulations are performed with at advective $\mathrm{CFL}=$ 0.3 , that is $\Delta t=\Delta t_{A} \approx 3.75 \mathrm{~s}$. The grid spacing is $\Delta x=$ $\Delta z=250 \mathrm{~m}$ and the trapezoidal time integrator is employed for the FC model. In agreement with published work (Restelli and Giraldo 2009), the Coriolis term is neglected here because of the small length of the channel.

Unlike the previous test cases, here the dynamics is chiefly wavelike rather than vertically buoyancy driven. Inertia-gravity waves develop in the horizontal direction (Fig. 10). As in the previous test case, only the FC contour plots are presented in Fig. 10 as the $\mathrm{PI}_{\rho, p}^{\mathrm{tc}}$ and $\mathrm{PI}_{\rho, p}$ plots are visually indistinguishable.

A quantitative comparison between the $\mathrm{FC}, \mathrm{PI}_{\rho, p}^{\mathrm{tc}}$, and $\mathrm{PI}_{\rho, p}$ results and the results of Restelli and Giraldo (2009) are reported on in Table 6. Maxima and minima of perturbations of velocity components, potential temperature, and Exner pressure at final time $T=3000 \mathrm{~s}$ are in line with published work.

The left panel of Fig. 11 shows a one-dimensional cut of the potential temperature perturbation at $z=5000 \mathrm{~m}$. As in the previous cases, the $\mathrm{PI}_{\rho, p}$ model displays a higher phase speed than the $\mathrm{PI}_{\rho, p}^{\mathrm{tc}}$ and FC models due to the neglect of pressure perturbations in the buoyancy term. The region of the leftmost crest is magnified in Fig. 11 to highlight the difference in the phase speed of

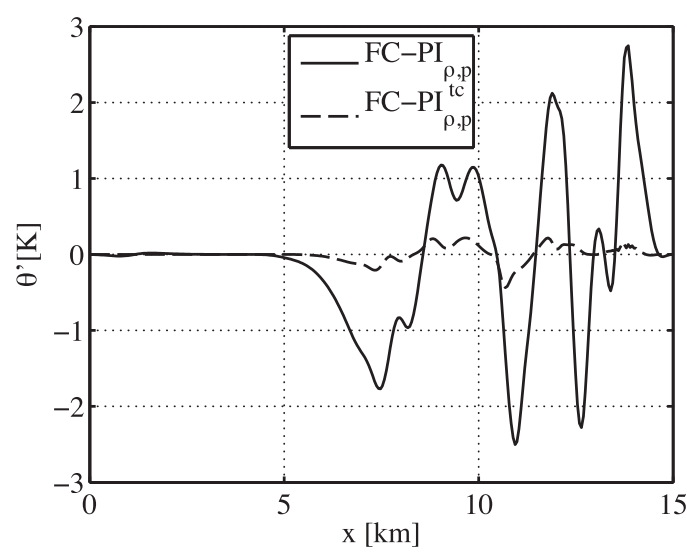

FIG. 9. Density current results: potential temperature perturbation at final time $T=900 \mathrm{~s}$. (left) A horizontal cut at height $z=1200 \mathrm{~m}$. (right) The difference from the FC profile of the $\mathrm{PI}_{\rho, p}^{\mathrm{tc}}$ profile (dashed line) and of the $\mathrm{PI}_{\rho, p}$ profile (solid line). 
TABLE 5. As in Table 3, but for density current and cuts at $z=$ $1200 \mathrm{~m}$ with respect to the FC cut as in Fig. 9.

\begin{tabular}{lccl}
\hline \hline & $E_{\mathrm{rel}}^{\mathrm{rms}}\left(\theta^{\prime}\right)$ & $E_{\mathrm{rel}}^{\max }\left(\theta^{\prime}\right)$ & \multicolumn{1}{c}{$E_{\mathrm{rel}}^{\max }\left(\theta_{\max }^{\prime}\right)$} \\
\hline $\mathrm{PI}_{\rho, p}^{\mathrm{tc}}-\mathrm{FC}$ & 0.046 & 0.090 & $1.93 \times 10^{-3}$ \\
$\mathrm{PI}_{\rho, p}-\mathrm{FC}$ & 0.441 & 0.584 & 0.026 \\
\hline
\end{tabular}

the $\mathrm{PI}_{\rho, p}$ model (dashed-dotted line) with respect to the $\mathrm{PI}_{\rho, p}^{\mathrm{tc}}$ model (asterisks) and the FC model (solid line).

The right panel of Fig. 11 shows the differences between the FC cut and the $\mathrm{PI}_{\rho, p}^{\mathrm{tc}}$ cut (dashed line) and between the FC cut and the $\mathrm{PI}_{\rho, p}$ cut (solid line). The amplitude of the difference is larger in the latter case due to the phase shift highlighted on the left panel. The result is quantified in Table 7 which shows relative RMS and max errors of the FC cut with respect to the $\mathrm{PI}_{\rho, p}^{\mathrm{tc}}$ and $\mathrm{PI}_{\rho, p}$ cuts. Relative $\mathrm{PI}_{\rho, p}-\mathrm{FC}$ errors are threefold those of $\mathrm{PI}_{\rho, p}^{\mathrm{tc}}-\mathrm{FC}$.

Finally, as in Restelli and Giraldo (2009) we define conservation errors as

$$
C_{\phi}=\frac{\left|\left(\phi_{\text {tot }}\right)_{T}-\left(\phi_{\text {tot }}\right)_{0}\right|}{\left(\phi_{\text {tot }}\right)_{0}}
$$

where $\phi_{\text {tot }}=\int_{\Omega} \phi d \mathbf{x}$ denotes the volumetric integral of $\phi$ in the domain $\Omega$. Subscripts 0 and $T$ denote initial and final time, respectively. We expect our scheme to conserve density $\rho$ and horizontal momentum density $\rho u$. Though our model does not conserve total energy $\rho E$, we report conservation scores for that variable, too. For the FC model, results for $P$ are also reported. Values of the conservation error for $\rho, \rho u, P$, and $\rho E$ are fairly low for the three model configurations (Table 8). Note, in Table 8 we define the total energy variable as

$$
E=\frac{1}{\rho} \frac{p}{\gamma-1}+\frac{\mathbf{v}^{2}}{2}+g z
$$

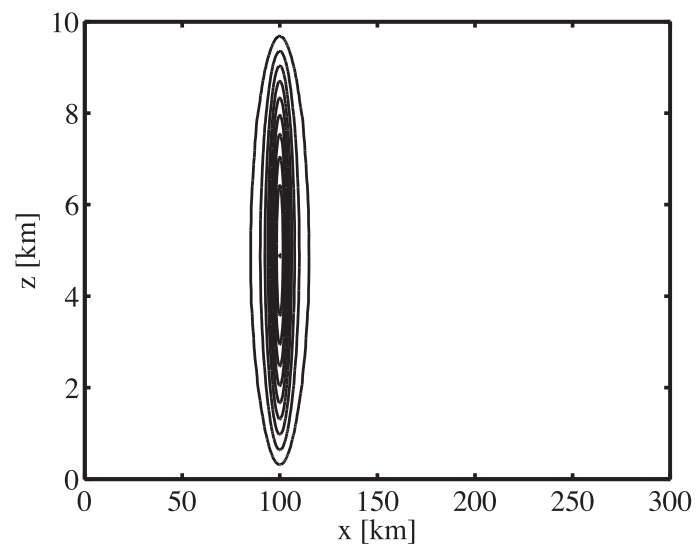

where $p=p_{0}$ in (46) for the $\mathrm{PI}_{\rho, p}^{\mathrm{tc}}$ and $\mathrm{PI}_{\rho, p}$ cases as shown in Klein and Pauluis (2012). Numerical analysis of the $P$ conservation is only meaningful for the FC model, since in the incompressible cases $P=P_{0}(z)$ holds.

\section{Discussion and conclusions}

We have presented a blended weakly compressible computational model with seamless access to thermodynamically consistent pseudo-incompressible dynamics, these two representing the limiting cases of a family of models depending on one parameter. For each member of the model family, the numerical discretization is the same up to certain weights in the stencil of the implicit corrector invoked to enable advection-based time steps in simulations of small to mesoscale systems.

This seamless and straightforward compressible-tosoundproof model transition can be realized in any flow solver that features the density and the mass-weighted potential temperature as prognostic variables for the thermodynamics, together with flux-based formulations of their determining equations. Weak checkerboard modes were observed in the runs of gravity-driven flows for very small time steps. We attribute them to the fact that the divergence of the cell-centered velocity is controlled in the second correction through a discrete elliptic problem derived from the linearized acoustic equations on the Arakawa B grid with a standard stencil. This grid arrangement allows for oscillatory modes with phase vectors pointing roughly along the grid diagonals (see Fig. 8 of Arakawa and Lamb 1977). These modes might be controllable by adopting a staggered grid arrangement (Arakawa $\mathrm{C}$ grid) or by adopting an inf-sup stable discretization of the elliptic operator on the B grid as in Vater and Klein (2009).

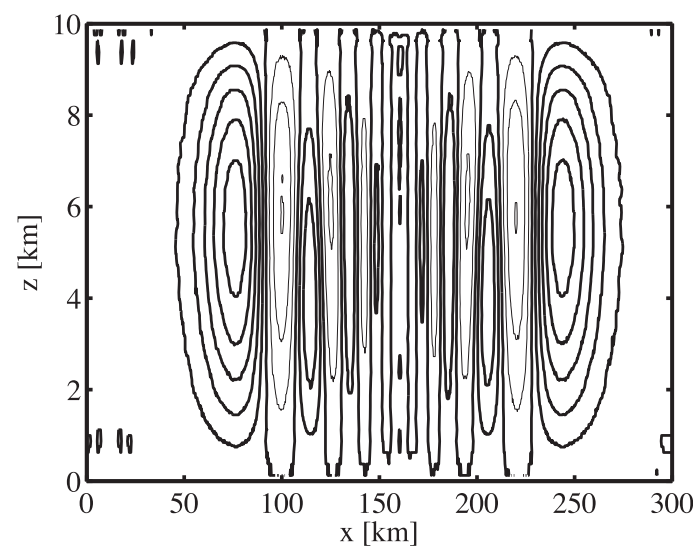

FIG. 10. Inertia-gravity wave results: potential temperature perturbation. (left) Initial data, contours every $10^{-3} \mathrm{~K}$. (right) FC result at $T=3000 \mathrm{~s}$, contours every $5 \times 10^{-4} \mathrm{~K}$ from -0.0015 to $0.003 \mathrm{~K}$. Thin lines denote negative contours. 
TABLE 6. Inertia-gravity wave results: maxima and minima of horizontal velocity $u$, vertical velocity $w$, potential temperature $\theta$, and Exner pressure $\pi=T \theta^{-1}$ perturbations at final time $T=3000 \mathrm{~s}$ in the present study and Restelli and Giraldo (2009, denoted by REF).

\begin{tabular}{lcccccccc}
\hline \hline & $u_{\max }^{\prime}$ & $u_{\min }^{\prime}$ & $w_{\max }^{\prime}$ & $w_{\min }^{\prime}$ & $\theta_{\max }^{\prime}$ & $\theta_{\min }^{\prime}$ & $\pi_{\max }^{\prime}$ & $\pi_{\min }^{\prime}$ \\
\hline $\mathrm{FC}$ & $1.054 \times 10^{-2}$ & $-1.060 \times 10^{-2}$ & $2.739 \times 10^{-3}$ & $-2.262 \times 10^{-3}$ & $2.808 \times 10^{-3}$ & $-1.526 \times 10^{-3}$ & $7.75 \times 10^{-7}$ & $-5.27 \times 10^{-7}$ \\
$\mathrm{PI}_{\rho, p}^{\mathrm{tc}}$ & $1.063 \times 10^{-2}$ & $-1.063 \times 10^{-2}$ & $2.645 \times 10^{-3}$ & $-2.424 \times 10^{-3}$ & $2.808 \times 10^{-3}$ & $-1.526 \times 10^{-3}$ & $1.18 \times 10^{-5}$ & $-6.56 \times 10^{-7}$ \\
$\mathrm{PI}_{\rho, p}$ & $1.365 \times 10^{-2}$ & $-1.362 \times 10^{-2}$ & $2.764 \times 10^{-3}$ & $-2.471 \times 10^{-3}$ & $2.930 \times 10^{-3}$ & $-1.709 \times 10^{-3}$ & $1.21 \times 10^{-5}$ & $-5.36 \times 10^{-7}$ \\
$\mathrm{REF}$ & $1.064 \times 10^{-2}$ & $-1.061 \times 10^{-2}$ & $2.877 \times 10^{-3}$ & $-2.400 \times 10^{-3}$ & $2.808 \times 10^{-3}$ & $-1.511 \times 10^{-3}$ & $9.11 \times 10^{-7}$ & $-7.13 \times 10^{-7}$ \\
\hline
\end{tabular}

The key observation enabling the blending is that, at least for an ideal gas with constant specific heat capacities, $\rho \theta$ is a function of pressure alone. Thus, the transport equation for $\rho \theta$ is equivalent to the pressure evolution equation and lends itself naturally for implicit pressure formulations. Once available, such a seamless framework can be used for a clean comparison of compressible and soundproof models that is not affected by sizeable differences between the respective model discretizations (see Smolarkiewicz and Dörnbrack 2008; Smolarkiewicz et al. 2014 for comparable arguments).

As a further potentially attractive application of such a modeling tool we suggest the filtering of unbalanced initial data. For given initial data, a matching pressure field and a related divergence correction that would guarantee a nearly sound-free subsequent evolution are generally not available. With a blended soundproofcompressible framework, one can generate accurate balanced pressure and velocity fields by running the model in soundproof mode for a few time steps and then making the transition to fully compressible over another few steps. This idea may also be transferred to other nearly balanced situations, such as hydrostatic and geostrophic, but exploring this is left for future work. In the framework of techniques for atmospheric data assimilation (Rabier 2005), the resulting ability of a computational model to manage and regularly embed new, unbalanced input in a balanced fashion and without invoking additional filtering procedures appears quite attractive. This capability can also be more generally useful when one has to map externally obtained data into a multidimensional finite-volume scheme as analyzed in Zingale et al. (2002).

Besides the aforementioned blending features, there are other noteworthy aspects of the scheme. First, we discretize the equations in full form without subtraction of a background state, maintaining accuracy by adopting a well-balanced discretization of the pressure gradient and gravity terms as discussed in Botta et al. (2004) and Klein (2009). Second, we cast the momentum equation in terms of pressure and density instead of the more common Exner pressure and potential temperature. The former choice guarantees conservation of momentum in the absence of external forces and increases flexibility with a view to implementing more general equations of state (Klein and Pauluis 2012).

Code performance was assessed in a number of configurations. The second-order accuracy of the scheme was verified on a smooth benchmark. Then, standard test cases consisting of buoyant thermal perturbations were considered, where our data confirmed no substantial difference between the compressible and pseudo-incompressible
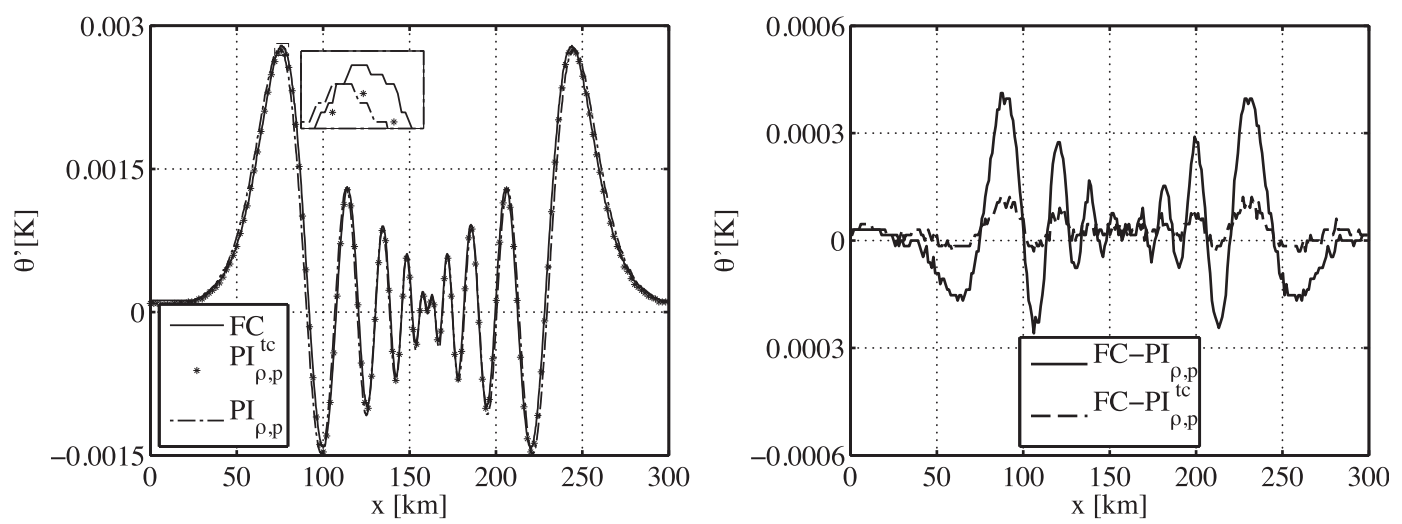

FIG. 11. Inertia-gravity wave results: potential temperature perturbation at final time. (left) A horizontal cut at height $z=5000 \mathrm{~m}$ for the FC model (solid line), the $\mathrm{PI}_{\rho, p}^{\mathrm{tc}}$ model (asterisks), and the $\mathrm{PI}_{\rho, p}$ model (dashed-dotted line). The region of the leftmost crest is magnified to highlight the higher phase speed of the $\mathrm{PI}_{\rho, p}$ model. (right) The difference from the FC cut for the $\mathrm{PI}_{\rho, p}^{\mathrm{tc}}$ cut (dashed line) and the $\mathrm{PI}_{\rho, p}$ cut (solid line). 
TABLE 7. Inertia-gravity wave results: relative root-meansquare error $E_{\mathrm{rel}}^{\mathrm{rms}}$ and maximum error $E_{\mathrm{rel}}^{\max }$ on potential temperature perturbation profile $\theta^{\prime}$ for the $\mathrm{PI}_{\rho, p}^{\mathrm{tc}}$ and $\mathrm{PI}_{\rho, p}$ cuts at $z=$ $5000 \mathrm{~m}$ with respect to the FC cut as in Fig. 11.

\begin{tabular}{lcc}
\hline & $E_{\mathrm{rel}}^{\mathrm{rms}}\left(\theta^{\prime}\right)$ & $E_{\mathrm{rel}}^{\max }\left(\theta^{\prime}\right)$ \\
\hline $\mathrm{PI}_{\rho, p}^{\mathrm{tc}}-\mathrm{FC}$ & 0.039 & 0.055 \\
$\mathrm{PI}_{\rho, p}-\mathrm{FC}$ & 0.132 & 0.16 \\
\hline
\end{tabular}

results. For the latter, including the linearized effect of pressure on density in the gravity term results not only in thermodynamic consistency (Klein and Pauluis 2012) but also in improved accuracy. Our findings are consistent with Davies et al. (2003) and Klein et al. (2010), thus confirming the validity of the pseudo-incompressible model at small- to mesoscales and for realistic stratifications.

As previously mentioned, we are planning to extend the present general stategy to include additional dominant balances relevant for larger-scale flows, specifically to the hydrostatic and geostrophic limits. This goal appears feasible in view of recent related work. For example, successful results have been achieved by Eulerian-semi-Lagrangian fluid solver (EULAG) model users (Prusa and Gutowski 2011; Szmelter and Smolarkiewicz 2011; Smolarkiewicz et al. 2014) with compressible, anelastic, and pseudo-incompressible models on the synoptic and planetary scales. Furthermore, alternatives have been explored to merge hydrostatic models with fully compressible (Janjic et al. 2001) or soundproof ones. Careful consideration will be needed to identify the correct large-scale limiting model in the light of recent suggestions of unified multiscale reduced models by Durran (2008), Arakawa and Konor (2009), and Konor (2014).

Acknowledgments. The authors thank Matthias Waidmann, Stephan Gerber, and Michael Oevermann for the fluid solver software this work is based upon. We thank the SAMRAI developers at Lawrence Livermore National Laboratory for their ingenious adaptive mesh refinement framework. Hilary Weller, Ronny Petrik, and another anonymous referee have helped us improving our work and presentation through numerous constructive comments. The same holds for Piotr Smolarkiewicz and Luca Bonaventura with whom we have also shared ideas along the way. We thank Deutsche Forschungsgemeinschaft for support through the Berlin Mathematical School, and Grants KL 611/ 23-1,2 ("MetStröm"). T. Benacchio and R. Klein greatly appreciated the opportunity to work for several weeks in the stimulating environment of the Newton Institute, Cambridge, United Kingdom, during the fall of 2012; and R. Klein acknowledges the support of the
TABLE 8. Inertia-gravity wave results: conservation errors for density, horizontal momentum density, $P$, and total energy density (see text for definitions) in the present study and in Restelli and Giraldo (2009, denoted by REF).

\begin{tabular}{llccc}
\hline \hline & \multicolumn{1}{c}{$C_{\rho}$} & \multicolumn{1}{c}{$C_{\rho \mathrm{u}}$} & $C_{P}$ & $C_{\rho \mathrm{E}}$ \\
\hline $\mathrm{FC}$ & $1.15 \times 10^{-9}$ & $8.05 \times 10^{-11}$ & $5.68 \times 10^{-9}$ & $1.98 \times 10^{-9}$ \\
$\mathrm{PI}_{\rho, p}^{\mathrm{tc}}$ & $6.77 \times 10^{-10}$ & $9.66 \times 10^{-10}$ & - & $3.99 \times 10^{-9}$ \\
$\mathrm{PI}_{\rho, p}$ & $8.90 \times 10^{-10}$ & $8.55 \times 10^{-10}$ & - & $4.21 \times 10^{-9}$ \\
$\mathrm{REF}$ & $1.67 \times 10^{-8}$ & $2.60 \times 10^{-7}$ & - & $1.64 \times 10^{-8}$ \\
\hline
\end{tabular}

National Center for Atmospheric Research, Boulder, Colorado, for financially supporting several research visits.

\section{APPENDIX}

\section{Details of the Numerical Scheme}

Here we elaborate on the aspects of the numerical scheme omitted in the main text.

\section{a. Predictor}

We use a second-order accurate, explicit two-stage strong stability-preserving Runge-Kutta method for time integration (Gottlieb et al. 2001). For the ordinary differential equation:

$$
\frac{d u}{d t}=L(u)
$$

where $L$ denotes a differential operator, the method reads

$$
\begin{aligned}
u^{(1)} & =u^{n}+\Delta t L\left(u^{n}\right), \\
u^{n+1} & =\frac{1}{2} u^{n}+\frac{1}{2} u^{(1)}+\frac{1}{2} \Delta t L\left[u^{(1)}\right],
\end{aligned}
$$

where $u^{(1)}$ denotes the first stage solution.

The spatial discretization is performed with a finitevolume approach (see, e.g., LeVeque 2002). Discrete variables are defined as approximations of cell averages set at cell centers, with the exception of dynamic pressure, set at cell nodes. The new cell-centered values are obtained from the old ones subtracting the net outflow flux at the boundaries and adding the contribution from the source term [see (21a)-(21c) in the main text].

The discretization of the fluxes is performed according to the following steps:

1) The velocity at the interfaces is determined by averaging the neighboring leftmost and rightmost cellcentered values $\mathbf{v}_{L}$ and $\mathbf{v}_{R}$ : 


$$
\mathbf{v}=\frac{1}{2}\left(\mathbf{v}_{L}+\mathbf{v}_{R}\right),
$$

where, for a second-order method, $\mathbf{v}_{L}$ and $\mathbf{v}_{R}$ have to be linearly reconstructed/limited. Considering the interface $\left(x_{i+1 / 2}, y_{j}\right)$, and omitting the subscript $j$ for simplicity, the reconstructed values of the horizontal velocity $u$ are

$u_{L}=u_{i}+\frac{1}{2} \psi\left(u_{i}-u_{i-1}, u_{i+1}-u_{i}\right)$,

$u_{R}=u_{i+1}-\frac{1}{2} \psi\left(u_{i+1}-u_{i}, u_{i+2}-u_{i+1}\right)$,

where

$$
\psi(a, b)=\frac{a+b}{2}
$$

for centered slopes. Our implementation features also an option for slope limiters, for which $\psi$ would have a different functional form. Upwind fluxes $F_{P}$ for the $P$ variable are computed by means of the obtained velocity:

$$
F_{P}=F_{P}^{+}+F_{P}^{-},
$$

where

$$
F_{P}^{+}=P_{L} \max (\mathbf{v}, 0), \quad F_{P}^{-}=P_{R} \min (\mathbf{v}, 0),
$$

and the subscripts $L$ and $R$ denote cell-centered leftmost and rightmost values of the variable.

2) Fluxes for the remaining quantities are referred to the carrier flux $P \mathbf{v}$ and derived using (A9) as

$$
F_{\phi}=F_{P}^{+} \phi_{L}+F_{P}^{-} \phi_{R},
$$

where $\phi \in\{1 / \theta, \mathbf{v} / \theta\}$. The contribution from the pressure term is incorporated in the momentum flux adding the pressure value at the center of the cell interface, obtained via average of the adjacent nodal values.

\section{b. Pressure update}

The nodal pressure update at the end of the time step proceeds as follows:

1) An auxiliary cell-centered pressure $p_{c}$ is computed from $P$ using the inverse of the equation of state in (2). The result is then interpolated to the nodes:

$$
p_{c}^{n+1}=\left(\frac{P^{n+1, * *}}{\rho_{\text {ref }} T_{\text {ref }}}\right)^{\gamma} p_{\text {ref }}-p_{\text {ref }}, \quad p_{c}^{n+1} \rightarrow p_{\mathrm{EOS}}^{n+1} .
$$

2) The obtained value is weighted with the old time level pressure update with the solution of (34) or (35), $\delta p$ :

$$
p^{n+1}=\alpha p_{\mathrm{EOS}}^{n+1}+(1-\alpha)\left(p^{n}+\delta p\right) .
$$

When the model runs in pseudo-incompressible mode with $\alpha=0$, the node-centered pressure increment $\delta p$ is summed to the old time level value. In compressible mode, with $\alpha=1$, the new nodal pressure is locked to $P$ imposing the equation of state at a discrete level.

Other solutions are possible and were tested. For example, as a pseudo-incompressible update, an interpolated value of the solution $\delta p_{c}$ of the first correction equation in (26) can be summed to the old time level pressure value. This was used in the thermal perturbations simulated with the fully compressible model initially run in pseudo-incompressible mode. In that case the solution of the second Poisson problem only serves as a correction to the momentum flux, (30), not as an update for the nodal pressure value.

\section{c. Time step choice}

The explicit time integration method adopted in the predictor step must be consistent with the CFL stability condition for advection (Courant et al. 1928), and a similar constraint for internal wave dynamics since these processes are handled explicitly in our scheme. In particular, we dynamically compute the time step size at each time loop according to

$$
\Delta t=\min \left(\Delta t_{I}, \Delta t_{A}, \Delta t_{B}\right),
$$

where $\Delta t_{I}$ is an externally imposed value of the time step. Here $\Delta t_{\mathrm{A}}$ is the advective time step:

$$
\Delta t_{A}=\frac{\mathrm{CFL} \Delta x}{\max _{\Omega}\left(\|\mathbf{v}\|_{2}\right)},
$$

where CFL $\leq 1$ and $\|\cdot\|_{2}$ is the discrete $L^{2}$ norm. Here $\Delta t_{\mathrm{B}}$ is a buoyancy-dependent time step:

$$
\Delta t_{B}=\mathrm{CFL} \sqrt{\frac{\Delta x \min _{\Omega} \theta}{g \max _{\Omega} \Delta \theta}},
$$

where $\max _{\Omega} \Delta \theta=\max _{\Omega} \theta-\min _{\Omega} \theta$ is the maximum potential temperature perturbation. Dynamically adaptive time stepping is standard in computational fluid dynamics and for two time level schemes its implementation is quite straightforward (LeVeque 2002).

\section{d. Well-balanced treatment of vertical pressure gradient and gravity term}

In the envisaged atmospheric applications, flow patterns arise as perturbations around a hydrostatically 
balanced state, where the vertical pressure gradient offsets the gravitational force:

$$
\frac{\partial p}{\partial z}=-\rho g .
$$

Therefore, an essential characteristic of a numerical method in this context is the capability of mimicking the hydrostatic balance at the discrete level. This means, for instance, that the numerical discretization should introduce no perturbations on an initially motionless atmospheric setting. The feature is especially nontrivial for models as the ones presented here whose analytical formulation relies on full variables, unlike other nonhydrostatic fully compressible models (e.g., Skamarock and Klemp 2008; Restelli and Giraldo 2009) wherein the unknowns are themselves perturbations around a background hydrostatically balanced reference state.

Here we adopt the approach of Botta et al. (2004), who describe the implementation of a discrete Archimedes' principle, and in the following we present the parts of our implementation tuned to take into account the hydrostatic balance.

\section{e. Initialization}

Since the problem is inherently one-dimensional, we focus on the vertical coordinate for the moment. First, let the initial data for pressure $p(z)$ and density $\rho(z)$ be given in the form of a homentropic or stably stratified atmosphere as in (37) or (42) above. Next:

- $p(z)$ is initialized in cell centers $z_{j}, j=1, \ldots, \mathcal{N}_{z}$ and nodes $z_{j-1 / 2}, j=1, \ldots, \mathcal{N}_{z}+1$ according to its analytical expression in (37) or (42);

- $\rho(z)$ is initialized at $z_{j}$ using a discretized form of (A16):

$$
\rho\left(z_{j}\right)=-\frac{1}{g \Delta z}\left[p\left(z_{j+1 / 2}\right)-p\left(z_{j-1 / 2}\right)\right], \quad j=1, \ldots, \mathcal{N}_{z},
$$

where $\Delta z$ is the vertical grid spacing.

\section{f. Predictor step}

The value of the pressure at the center of the cell face needed for the momentum flux computation in (19b) is computed as follows:

$$
\begin{aligned}
p\left(z_{j}\right)= & \frac{1}{2}\left\{p\left(z_{j+1 / 2}\right)+p\left(z_{j-1 / 2}\right)\right. \\
& \left.-g\left[2 f\left(z_{j}\right)-f\left(z_{j+1 / 2}\right)-f\left(z_{j-1 / 2}\right)\right]\right\}
\end{aligned}
$$

for $j=1, \ldots, \mathcal{N}_{z}$, where

$$
f(z)=\int_{0}^{z} \rho\left(z^{\prime}\right) d z^{\prime}
$$

and the square bracket in (A18) represents a hydrostatic modification of the simple average.

\section{g. Boundary conditions}

The so-called solid wall boundary conditions are adjusted to take into account hydrostatic balance. As customary in finite-differences and finite-volume codes (LeVeque 2002), we implement fully reflecting boundaries using "ghost cells." The strategy involves attaching two dummy cells to the boundary in which the value of all the variables except for the normal velocity is mirrored from the two innermost cells, whereas the normal velocity value is taken with the opposite sign.

We modify the process for the mirrored variables in that we retrieve in the ghost cells the hydrostatically consistent values. For instance, for the pressure in the first lower ghost cell (cell 0) we have the following:

$$
p\left(z_{0}\right)=p\left(z_{1}\right)+g \int_{z_{0}}^{z_{1}} \rho(z) d z
$$

and similar expressions hold for the upper values.

\section{h. Final locking of pressure and P variables}

The third modification involves the interpolation from nodes to cell centers or vice versa, which in the case without gravity is a standard linear interpolation. Here, a correction taking into account hydrostaticity is introduced. In particular, for the cell-to-node interpolation used in the pressure update in (A11) after the second correction step:

- For the lower boundary nodes:

$$
p\left(x_{i+1 / 2}, z_{1 / 2}\right)=0.5\left(p_{\mathrm{NW}}+p_{\mathrm{NE}}\right), \quad \forall i=1, \ldots, \mathcal{N}_{x},
$$

where $p_{\mathrm{NW}}$ and $p_{\mathrm{NE}}$ denote the pressure values obtained with analytical integration downward from the hydrostatic pressure values in the adjacent top-left and top-right cell, respectively.

- For the upper boundary nodes:

$$
p\left(x_{i+1 / 2}, z_{\mathcal{N}_{z}+1 / 2}\right)=0.5\left(p_{\mathrm{SW}}+p_{\mathrm{SE}}\right), \quad \forall \quad i=1, \ldots, \mathcal{N}_{x},
$$

where $p_{\mathrm{SW}}$ and $p_{\mathrm{SE}}$ denote the pressure values obtained with analytical integration upward from the hydrostatic pressure values in the adjacent bottom-left and bottom-right cell, respectively.

- For the internal nodes: 


$$
\begin{gathered}
p\left(x_{i+1 / 2}, z_{j+1 / 2}\right)=0.25\left(p_{\mathrm{SW}}+p_{\mathrm{SE}}+p_{\mathrm{NW}}+p_{\mathrm{NE}}\right), \\
\forall i=1, \ldots, \mathcal{N}_{x}, \quad j=1, \ldots, \mathcal{N}_{z}-1 .
\end{gathered}
$$

Finally, we remark that issues due to neglect of hydrostatic balance at the discrete level manifest less in the incompressible than in the fully compressible version of our method. In the former, small spurious perturbations due to inexact balancing, for instance, at the boundary are projected away in the correction step, while in the latter $P$ and pressure are locked through the equation of state, thus requiring a careful adjustment.

\section{REFERENCES}

Achatz, U., R. Klein, and F. Senf, 2010: Gravity waves, scale asymptotics and the pseudo-incompressible equations. J. Fluid Mech., 663, 120-147, doi:10.1017/S0022112010003411.

Almgren, A. S., J. B. Bell, P. Colella, L. H. Howell, and M. L. Welcome, 1998: A conservative adaptive projection method for the variable density incompressible NavierStokes equations. J. Comput. Phys., 142, 1-46, doi:10.1006/ jcph.1998.5890.

$\longrightarrow,-$ C. A. Rendleman, and M. Zingale, 2006a: Low Mach number modeling of type Ia supernovae. I. Hydrodynamics. Astrophys. J., 637, 922-936, doi:10.1086/498426.

,,,--- and $-2006 \mathrm{~b}$ : Low Mach number modeling of type Ia supernovae. II. Energy evolution. Astrophys. J., 649, 927-938, doi:10.1086/507089.

Arakawa, A., and V. R. Lamb, 1977: Computational design of the basic dynamical processes of the UCLA general circulation model. Methods Comput. Phys., 17, 173-265.

— , and C. S. Konor, 2009: Unification of the anelastic and quasihydrostatic systems of equations. Mon. Wea. Rev., 137, 710726, doi:10.1175/2008MWR2520.1.

Bannon, P. R., 1996: On the anelastic approximation for a compressible atmosphere. J. Atmos. Sci., 53, 3618-3628, doi:10.1175/ 1520-0469(1996)053<3618:OTAAFA>2.0.CO;2.

Bell, J., L. Howell, and P. Colella, 1991: An efficient second-order projection method for viscous incompressible flow. Proc. 10th AIAA Computational Fluid Dynamics Conf., Honolulu, HI, AIAA, Vol. 360, doi:10.2514/6.1991-1560.

Bijl, H., and P. Wesseling, 1998: A unified method for computing incompressible and compressible flows in boundary-fitted coordinates. J. Comput. Phys., 141, 153-173, doi:10.1006/ jcph.1998.5914.

Bonaventura, L., 2000: A semi-implicit semi-Lagrangian scheme using the height coordinate for a nonhydrostatic and fully elastic model of atmospheric flows. J. Comput. Phys., 158, 186213, doi:10.1006/jcph.1999.6414.

Botta, N., R. Klein, S. Langenberg, and S. Lützenkirchen, 2004: Well balanced finite volume methods for nearly hydrostatic flows. J. Comput. Phys., 196, 539-565, doi:10.1016/ j.jcp.2003.11.008.

Brdar, S., M. Baldauf, A. Dedner, and R. Klöfkorn, 2013: Comparison of dynamical cores for NWP models: Comparison of COSMO and Dune. Theor. Comput. Fluid Dyn., 27, 453-472, doi:10.1007/s00162-012-0264-Z.
Casulli, V., and D. Greenspan, 1984: Pressure correction method for the numerical solution of transient compressible fluid flows. Int. J. Numer. Methods Fluids, 4, 1001-1012, doi:10.1002/ fld.1650041102.

Courant, R., K. Friedrichs, and H. Lewy, 1928: Über die partiellen Differenzengleichungen der mathematischen Physik (On the partial difference equations of mathematical physics). Math. Ann., 100, 32-74, doi:10.1007/BF01448839.

Cullen, M. J. P., 2007: Modelling atmospheric flows. Acta Numer., 16, 67-154, doi:10.1017/S0962492906290019.

Davies, T., A. Staniforth, N. Wood, and J. Thuburn, 2003: Validity of anelastic and other equation sets as inferred from normalmode analysis. Quart. J. Roy. Meteor. Soc., 129, 2761-2775, doi:10.1256/qj.02.1951.

— M. J. P. Cullen, A. J. Malcolm, M. H. Mawson, A. Staniforth, A. White, and N. Wood, 2005: A new dynamical core for the Met Office's global and regional modelling of the atmosphere. Quart. J. Roy. Meteor. Soc., 131, 1759-1782, doi:10.1256/ qj.04.101.

Dukowicz, J. K., 2013: Evaluation of various approximations in ocean and atmospheric modeling based on an exact treatment of gravity wave dispersion. Mon. Wea. Rev., 141, 4487-4506, doi:10.1175/MWR-D-13-00148.1.

Durran, D. R., 1989: Improving the anelastic approximation. J. Atmos. Sci., 46, 1453-1461, doi:10.1175/1520-0469(1989)046<1453: ITAA $>2.0 . \mathrm{CO} ; 2$.

_ 2008: A physically motivated approach for filtering acoustic waves from the equations governing compressible stratified flow. J. Fluid Mech., 601, 365-379, doi:10.1017/ S0022112008000608.

_ , and P. N. Blossey, 2012: Implicit-explicit multistep methods for fast-wave-slow-wave problems. Mon. Wea. Rev., 140, 1307-1325, doi:10.1175/MWR-D-11-00088.1.

Falgout, R. D., J. E. Jones, and U. M. Yang, 2006: The design and implementation of hypre, a library of parallel high performance preconditioners. Numerical Solution of Partial Differential Equations on Parallel Computers, A. Bruaset and A. Tveito, Eds., Lecture Notes in Computational Science and Engineering, Vol. 51, Springer, 267-294.

Gatti-Bono, C., and P. Colella, 2006: An anelastic allspeed projection method for gravitationally stratified flows. J. Comput. Phys., 216, 589-615, doi:10.1016/j.jcp.2005.12.017.

Giraldo, F. X., J. F. Kelly, and E. M. Costantinescu, 2013: Implicitexplicit formulations of a three-dimensional nonhydrostatic unified model of the atmosphere (NUMA). SIAM J. Sci. Comput., 35, B1162-B1194, doi:10.1137/120876034.

Gottlieb, S., C.-W. Shu, and E. Tadmor, 2001: Strong stabilitypreserving high-order time discretization methods. SIAM Rev., 43, 89-112, doi:10.1137/S003614450036757X.

Haack, J., S. Jin, and J.-G. Liu, 2012: An all-speed asymptoticpreserving method for the isentropic Euler and Navier-Stokes equations. Commun. Comput. Phys., 12, 955-980.

Hohenegger, C., and C. Schär, 2007: Predictability and error growth dynamics in cloud-resolving models. J. Atmos. Sci., 64, 4467-4478, doi:10.1175/2007JAS2143.1.

Hornung, R. D., A. M. Wissink, and S. R. Kohn, 2006: Managing complex data and geometry in parallel structured AMR applications. Eng. Comput., 22, 181-195, doi:10.1007/ s00366-006-0038-6.

Hortal, M., 2002: The development and testing of a new two-timelevel semi-Lagrangian scheme (SETTLS) in the ECMWF forecast model. Quart. J. Roy. Meteor. Soc., 128, 1671-1687, doi:10.1002/qj.200212858314. 
Janjic, Z. I., J. P. Gerrity, and S. Nickovic, 2001: An alternative approach to nonhydrostatic modeling. Mon. Wea. Rev., 129, 1164-1178, doi:10.1175/1520-0493(2001)129<1164: AAATNM $>2.0 . \mathrm{CO} ; 2$.

Jebens, S., O. Knoth, and R. Weiner, 2009: Explicit two-step peer methods for the compressible Euler equations. Mon. Wea. Rev., 137, 2380-2392, doi:10.1175/2008MWR2671.1.

,$- \ldots$, and -2011 : Partially implicit peer methods for the compressible Euler equations. J. Comput. Phys., 230, 49554974, doi:10.1016/j.jcp.2011.03.015.

Kadioglu, S. Y., R. Klein, and M. L. Minion, 2008: A fourth-order auxiliary variable projection method for zero-Mach number gas dynamics. J. Comput. Phys., 227, 2012-2043, doi:10.1016/ j.jcp.2007.10.008.

Kelly, J. F., and F. X. Giraldo, 2012: Continuous and discontinuous Galerkin methods for a scalable threedimensional nonhydrostatic atmospheric model: Limitedarea mode. J. Comput. Phys., 231, 7988-8008, doi:10.1016/ j.jcp.2012.04.042.

Klein, R., 2000: Asymptotic analyses for atmospheric flows and the construction of asymptotically adaptive numerical methods. Z. Angew. Math. Mech., 80, 765-777, doi:10.1002/ 1521-4001(200011)80:11/12<765::AID-ZAMM765>3.0.CO;2-1.

- 2009: Asymptotics, structure, and integration of sound-proof atmospheric flow equations. Theor. Comput. Fluid Dyn., 23, 161-195, doi:10.1007/s00162-009-0104-y.

_ 2010: Scale-dependent models for atmospheric flows. Annu. Rev. Fluid Mech., 42, 249-274, doi:10.1146/ annurev-fluid-121108-145537.

— a pseudoincompressible approximation for general equations of state. J. Atmos. Sci., 69, 961-968, doi:10.1175/ JAS-D-11-0110.1.

— N. Botta, L. Hofmann, A. Meister, C. Munz, S. Roller, and T. Sonar, 2001: Asymptotic adaptive methods for multiscale problems in fluid mechanics. J. Eng. Math., 39, 261-343, doi:10.1023/A:1004844002437.

_ , U. Achatz, D. Bresch, O. M. Knio, and P. Smolarkiewicz, 2010: Regime of validity of soundproof atmospheric flow models. J. Atmos. Sci., 67, 3226-3237, doi:10.1175/2010JAS3490.1.

_- T. Benacchio, and W. P. O'Neill, 2014: Using the sound-proof limit for balanced data initialization. Proc. ECMWF Seminar on Numerical Methods, Reading, United Kingdom, ECMWF, 227-236

Konor, C. S., 2014: Design of a dynamical core based on the nonhydrostatic "unified system" of equations. Mon. Wea. Rev., 142, 364-385, doi:10.1175/MWR-D-13-00187.1.

Kwatra, N., J. Su, J. T. Grétarsson, and R. Fedkiw, 2009: A method for avoiding the acoustic time step restriction in compressible flow. J. Comput. Phys., 228, 4146-4161, doi:10.1016/ j.jcp.2009.02.027.

LeVeque, R. J., 2002: Finite Volume Methods for Hyperbolic Problems. Cambridge University Press, $580 \mathrm{pp}$.

Lipps, F. B., and R. S. Hemler, 1982: A scale analysis of deep moist convection and some related numerical calculations. J. Atmos. Sci., 39, 2192-2210, doi:10.1175/1520-0469(1982)039<2192: ASAODM $>2.0 . \mathrm{CO} ; 2$.

Munz, C.-D., S. Roller, R. Klein, and K. Geratz, 2003: The extension of incompressible flow solvers to the weakly compressible regime. Comput. Fluids, 32, 173-196, doi:10.1016/ S0045-7930(02)00010-5.

Nonaka, A., A. S. Almgren, J. B. Bell, M. J. Lijewski, C. M. Malone, and M. Zingale, 2010: MAESTRO: An adaptive low Mach number hydrodynamics algorithm for stellar flows. Astrophys. J. Suppl. Ser., 188, 358, doi:10.1088/0067-0049/188/2/358.

Prusa, J. M., and W. J. Gutowski, 2011: Multi-scale waves in soundproof global simulations with EULAG. Acta Geophys., 59, 1135-1157, doi:10.2478/s11600-011-0050-0.

Rabier, F., 2005: Overview of global data assimilation developments in numerical weather-prediction centres. Quart. J. Roy. Meteor. Soc., 131, 3215-3233, doi:10.1256/qj.05.129.

Reisner, J. M., A. Mousseau, A. A. Wyszogrodzki, and D. A. Knoll, 2005: An implicitly balanced hurricane model with physicsbased preconditioning. Mon. Wea. Rev., 133, 1003-1022, doi:10.1175/MWR2901.1.

Restelli, M., and F. X. Giraldo, 2009: A conservative discontinuous Galerkin semi-implicit formulation for the NavierStokes equations in nonhydrostatic mesoscale modeling. SIAM J. Sci. Comput., 31, 2231-2257, doi:10.1137/ 070708470.

Schneider, T., N. Botta, K. J. Geratz, and R. Klein, 1999: Extension of finite volume compressible flow solvers to multi-dimensional, variable density zero Mach number flows. J. Comput. Phys., 155, 248-286, doi:10.1006/jcph.1999.6327.

Skamarock, W. C., and J. B. Klemp, 1994: Efficiency and accuracy of the Klemp-Wilhelmson time-splitting technique. Mon. Wea. Rev., 122, 2623-2630, doi:10.1175/1520-0493(1994)122<2623: EAAOTK $>2.0 . \mathrm{CO} ; 2$.

—, and —, 2008: A time-split nonhydrostatic atmospheric model for weather research and forecasting applications. J. Comput. Phys., 227, 3465-3485, doi:10.1016/j.jcp.2007.01.037.

Smith, J. W., and P. R. Bannon, 2008: A comparison of compressible and anelastic models of deep dry convection. Mon. Wea. Rev., 136, 4555-4571, doi:10.1175/ 2008MWR2343.1.

Smolarkiewicz, P. K., and A. Dörnbrack, 2008: Conservative integrals of adiabatic Durran's equations. Int. J. Numer. Methods Fluids, 56, 1513-1519, doi:10.1002/fld.1601.

—_, and P. Charbonneau, 2013: EULAG, a computational model for multiscale flows: An MHD extension. J. Comput. Phys., 236, 608-623, doi:10.1016/j.jcp.2012.11.008.

—, C. Kühnlein, and N. Wedi, 2014: A consistent framework for discrete integrations of soundproof and compressible PDEs of atmospheric dynamics. J. Comput. Phys., 263, 185205, doi:10.1016/j.jcp.2014.01.031.

Straka, J. M., R. B. Wilhelmson, L. J. Wicker, J. R. Anderson, and K. K. Droegemeier, 1993: Numerical solutions of a nonlinear density current: A benchmark solution and comparisons. Int. J. Numer. Methods Fluids, 17, 1-22, doi:10.1002/ fld.1650170103.

Szmelter, J., and P. K. Smolarkiewicz, 2011: An edge-based unstructured mesh framework for atmospheric flows. Comput. Fluids, 46, 455-460, doi:10.1016/j.compfluid.2010.10.020.

Vasil, G. M., D. Lecoanet, B. P. Brown, T. S. Wood, and E. G. Zweibel, 2013: Energy conservation and gravity waves in sound-proof treatments of stellar interiors. II. Lagrangian constrained analysis. Astrophys. J., 773, 169, doi:10.1088/ 0004-637X/773/2/169.

Vater, S., 2013: A multigrid-based multiscale numerical scheme for shallow water flows at low Froude number. Ph.D. thesis, Freie Universität Berlin, Berlin, Germany, 179 pp. [Available online at http://edocs.fu-berlin.de/diss/receive/FUDISS_thesis_ 000000093897.]

, and R. Klein, 2009: Stability of a Cartesian grid projection method for zero Froude number shallow water flows. Numer. Math., 113, 123-161, doi:10.1007/s00211-009-0224-8. 
Weller, H., and A. Shahrokhi, 2014: Curl-free pressure gradients over orography in a solution of the fully compressible Euler equations with implicit treatment of acoustic and gravity waves. Mon. Wea. Rev., 142, 4439-4457, doi:10.1175/MWR-D-14-00054.1.

Wong, M., W. C. Skamarock, P. H. Lauritzen, J. B. Klemp, and R. B. Stull, 2014: A compressible nonhydrostatic cell-integrated semi-Lagrangian semi-implicit solver (CSLAM-NH) with consistent and conservative transport. Mon. Wea. Rev., 142, 1669-1687, doi:10.1175/MWR-D-13-00210.1.

Wood, N., and Coauthors, 2014: An inherently massconserving semi-implicit semi-Lagrangian discretization of the deep-atmosphere global non-hydrostatic equations. Quart. J. Roy. Meteor. Soc., 140, 1505-1520, doi:10.1002/ qj. 2235.

Zängl, G., D. Reinert, P. Rípodas, and M. Baldauf, 2014: The ICON (ICOsahedral Non-hydrostatic) modelling framework of DWD and MPI-M: Description of the non-hydrostatic dynamical core. Quart. J. Roy. Meteor. Soc., doi:10.1002/ qj.2378, in press.

Zingale, M., and Coauthors, 2002: Mapping initial hydrostatic models in Godunov codes. Astrophys. J. Suppl. Ser., 143, 539565, doi:10.1086/342754. 